\title{
Heavy-element Rydberg transition line emission from the post-giant-evolution star HD 101584
}

\author{
H. Olofsson ${ }^{1}$, J.H. Black ${ }^{1}$, T. Khouri ${ }^{1}$, W.H.T. Vlemmings ${ }^{1}$, E.M.L. Humphreys ${ }^{2}$, M. Lindqvist ${ }^{1}$, M. Maercker ${ }^{1}$, \\ L. Nyman ${ }^{3}$, S. Ramstedt ${ }^{4}$, and D. Tafoya ${ }^{1}$
}

\author{
1 Dept of Space, Earth and Environment, Chalmers Univ. of Technology, Onsala Space Observatory, SE-43992 Onsala, Sweden \\ e-mail: hans.olofsson@chalmers.se \\ 2 ESO, Karl-Schwarzschild-Str. 2, D-85748 Garching bei München, Germany \\ 3 ESO, Alonso de Cordova 3107, Vitacura, Santiago, Chile \\ ${ }^{4}$ Dept of Physics and Astronomy, Uppsala University, Box 516, SE-75120 Uppsala, Sweden
}

Received 12 February 2021 / Accepted 20 April 2021

\begin{abstract}
Context. We report the detection of two lines at millimetre wavelengths towards the immediate surroundings of the post-giant and most likely post-common-envelope star HD 101584 using high-angular-resolution ALMA observations. The circumstellar environment of this object is rich in different molecular species, but we find no viable identifications in terms of molecular lines.

Aims. We aim to determine whether or not these lines can be attributed to the Rydberg transitions - X $30 \alpha$ and X26 $\alpha-$ of neutral atoms of elements heavier than carbon.

Methods. A simple model in strict local thermodynamic equilibrium for a warm-gas environment of the moderate-temperature star $\left(T_{\text {eff }} \approx 8500 \mathrm{~K}\right)$ was constructed to corroborate our findings. A geometrically thin, disc-like geometry seen face-on was chosen and a distance of $1 \mathrm{kpc}$.

Results. The observed flux densities of the lines and the continuum at 232 and $354 \mathrm{GHz}$ can be reproduced using $\approx 10^{-3} M_{\odot}$ of gas at a temperature of $\approx 2800 \mathrm{~K}$ and a hydrogen density of $\approx 10^{12} \mathrm{~cm}^{-3}$, assuming solar abundances for the elements. The gas lies within a distance of about 5 au from the star (assuming a distance of $1 \mathrm{kpc}$ ). The ionisation fraction is low, $\approx 3 \times 10^{-5}$. The origin of such a region is not clear, but it may be related to a common-envelope-evolution phase. With these conditions, the line emissions are dominated by Rydberg transitions within the stable isotopes of $\mathrm{Mg}$. A turbulent velocity field in the range $5.5-7.5 \mathrm{~km} \mathrm{~s}^{-1}$ is required to fit the Gaussian line shapes. An upper limit to the average magnetic field in the line-emitting region of $1 \mathrm{G}$ is set using the Zeeman effect in these lines.

Conclusions. We speculate that Rydberg transitions of heavy elements may be an interesting probe for the close-in environments of other moderate-temperature objects like AGB stars, red supergiants, yellow hypergiants, and binaries of various types.
\end{abstract}

Key words. circumstellar matter - Stars: individual: HD101584 - Stars: AGB and post-AGB - Radio lines: stars

\section{Introduction}

As pointed out already by Bohr (1920), in the limit of large quantum numbers $n$, all atomic spectra appear 'hydrogenic' with the ionisation energy of a single electron far away from the ionic core well described by the classical Rydberg formula, $E_{\mathrm{n}} \propto n^{-2}$. Such highly excited atoms occur frequently in different astronomical objects where conditions are favourable; for instance, at low densities and in intense photo-ionising fields. The theory of Rydberg transitions in the cosmic nebular environment was first developed by Menzel and collaborators in a series of papers starting in 1937 (e.g. Menzel 1937). The possibility to detect such cosmic lines at radio wavelengths was first discussed by van de Hulst (1945) and clarified quantitatively by Kardashev (1959), specifically in the tenuous nebular medium which is exposed to intense UV light, where highly excited Rydberg states are populated by radiative recombination of the parent ion and a free electron; hence the name, (radio) 'recombination' lines.

The first detections of cosmic radio recombination lines from hydrogen were made by Dravskikh \& Dravskikh (1964) and Höglund \& Mezger (1965) towards H II regions. Subsequently, recombination lines from helium were detected towards H II regions, and carbon lines were detected from a cooler medium sur- rounding H II regions (Lilley et al. 1966, Pankonin et al. 1977). Chaisson et al. (1972) found a weak feature on the blueshifted side of the $\mathrm{C} 158 \alpha$ line, and identified it as due to one or a combination of the heavy element species $\mathrm{Mg}, \mathrm{Si}, \mathrm{S}$, and Fe. Safe identification of the carrier is often hampered by the fact that the line separations - only due to the differences in reduced massof elements heavier than carbon are smaller than the line widths. However, in the cold $\rho$ Oph dark cloud, Chaisson (1975) managed to identify a sulphur recombination line. Recombination lines have now been detected also towards other ionising sources like planetary nebulae (Roelfsema et al. 1991; Bachiller et al. 1992, Sánchez Contreras et al. 2017), luminous blue variables like $\eta$ Car (Cox et al. 1995), and young stellar objects (MartinPintado et al. 1989; Thum et al. 2013). Comprehensive reviews of recombination lines are given in Brown et al. (1978) and Gordon \& Sorochenko (2002). Also the Sun has been detected in Rydberg transitions at submillimetre (submm; $\mathrm{H}$ and possibly $\mathrm{Mg}$ (Clark et al. 2000a b) ) and mid-infrared (Mg and Al (Murcray et al. 1981; Brault \& Noyes 1983, Chang \& Noyes 1983)) wavelengths. Here the conditions are different, and a different approach is required to describe the excitation (Carlsson \& Rutten 1992); therefore, using the nomenclature of Rydberg transi- 
tion lines (RTLs) rather than recombination lines is more appropriate.

Here we present the detection of spectral lines at about 232 and $354 \mathrm{GHz}$ towards the moderate-temperature, low-mass star HD 101584 using the Atacama Large Millimeter/submillimeter Array (ALMA). We argue that these lines are RTLs of neutral atoms of elements heavier than carbon. A simple model in strict local thermal equilibrium (LTE) is used to estimate the viability of this proposal, and to estimate the physical characteristics of the line-emitting region and identify the most likely carrier (or carriers) of the lines.

\section{HD 101584}

HD 101584 is a binary system with a significant infrared excess. For the primary star, we adopt a spectral type of A6Ia and effective temperature of $8500 \mathrm{~K}$; see Kipper (2005) who determined the temperature from spectral synthesis of high-resolution optical spectra. It has been difficult to establish whether its current state represents evolution beyond the asymptotic giant branch (AGB) or the red giant branch (RGB). It was originally classified as a post-AGB star (Parthasarathy \& Pottasch 1986; Bakker et al. 1996), but Olofsson et al. (2019) classified it as a post-RGB star, the consequence of premature termination of the RGB caused by common-envelope (CE) evolution that ended before the two stars merged.

Olofsson et al. (2019) studied the circumstellar environment of HD 101584 in detail using ALMA. This object is morphologically and kinematically complex, consisting of a bipolar highvelocity outflow (seen almost pole-on) surrounded by an hourglass structure, an equatorial density enhancement (seen almost face-on), and a central compact source (whose geometry and orientation are unknown). A total of 12 different molecular species (not counting isotopologues) have been identified in the circumstellar medium. Here we recapitulate our present knowledge of the near environment of HD 101584. For more information on this source, see Olofsson et al. (2019) and references therein.

Many of the molecular line emissions observed using ALMA show a compact component that coincides in space with the position of HD 101584, the central compact source (CCS) in the nomenclature of Olofsson et al. (2019); detected molecular species are $\mathrm{CO}, \mathrm{SiO}, \mathrm{SiS}, \mathrm{CS}, \mathrm{SO}, \mathrm{SO}_{2}, \mathrm{OCS}$, and $\mathrm{H}_{2} \mathrm{~S}$. The emission from this component is partially resolved, and its estimated mass is $\approx 0.03[D / 1 \mathrm{kpc}]^{2} M_{\odot}$, which corresponds to $\approx 7 \%$ of the total circumstellar gas mass. The angular size varies with the observed molecular transition, but lies in the range 0 '! $1-00^{\prime \prime} 15$ (FWHM of Gaussian fit to the brightness distribution deconvolved with the synthesised beam), that is, within $\approx 75[D / 1 \mathrm{kpc}]$ au of HD101584. The morphology of this component has not been determined, except for the innermost structure observed with the VLTI/PIONIER instrument. Kluska et al. (2020) detected an almost circular structure interpreted to be the sublimation rim of a circumbinary dust disc seen close to face on. The radius of this ring is about $2[D / 1 \mathrm{kpc}]$ au. The lines discussed in this paper most likely come from a region within a radius of about $5[D / 1 \mathrm{kpc}]$ au from the binary. The binary separation is estimated to be about $0.5 \mathrm{au}$.

The systemic velocity of HD 101584 is estimated to be $41.5 \pm 0.15 \mathrm{~km} \mathrm{~s}^{-1}$ (in LSR scale). We have derived the average velocity from Gaussian fits to six ALMA lines that show strong emission from the CCS: $\mathrm{CO}(2-1), \mathrm{C}^{18} \mathrm{O}(2-1), \mathrm{SO}\left(5_{5}-4_{4}\right)$, $\mathrm{SO}\left(5_{6}-4_{5}\right), \mathrm{SO}_{2}\left(16_{3,13}-16_{2,14}\right)$, and $\mathrm{H}_{2} \mathrm{~S}\left(2_{20}-2_{11}\right)$; see Olofsson et al. (2019). The systemic velocity is crucial for deriving the rest frequencies of the lines discussed in this paper.
The parallax of HD 101584 in the second Gaia data release suggests a distance as large as $2 \mathrm{kpc}$ (Gaia Collaboration et al. 2016, 2018). The third Gaia data release maintains this distance, but the excess noise required in the fitting process has increased (Gaia Collaboration et al. 2020). However, as argued by Olofsson et al. (2019) there are reasons to suspect that this distance is not correct, the primary one being that the estimated angular distance between HD 101584 and its companion is comparable to the parallax. Consequently, we follow their adopted procedure of presenting all results for a nominal distance of $D=1.0 \mathrm{kpc}$. From a fit to the spectral energy distribution, Olofsson et al. (2019) derived a luminosity and radius of the star of $1600 L_{\odot}$ and $19 R_{\odot}$, respectively, at $1 \mathrm{kpc}$, based on the assumption that the radiation of the infrared excess is not isotropic.

\section{Observations}

\subsection{ALMA data}

The ALMA data were obtained during cycles 1 (May 2014, TA1), 3 (October 2015, TA2; September 2016, TA3), and 6 (November 2018, TA4) with 35 to 46 antennas of the $12 \mathrm{~m}$ main array in one frequency setting in each of Band 6 (TA1-TA3; $232 \mathrm{GHz}$ ) and Band 7 (TA4; $354 \mathrm{GHz}$ ). In both settings, the data set contains four $1.875 \mathrm{GHz}$ spectral windows with 1920 channels each, except for one spectral window in the cycle 3 data that has 960 channels covering $1.875 \mathrm{GHz}$. The final velocity resolutions are 3.0 and $1.0 \mathrm{~km} \mathrm{~s}^{-1}$ for the Band 6 and 7 data, respectively. The baselines range from 13 to $16196 \mathrm{~m}$ for band 6 and 15 to $1398 \mathrm{~m}$ for Band 7. This means a highest angular resolution of about $00^{\prime \prime} 03$ and $00^{\prime \prime 2}$ for the two bands, respectively. Bandpass calibration was performed on J1107-4449 (TA1, TA2, TA3) and J1037-2934 and J0635-7516 (TA4), and gain calibration on J1131-5818 (TA1), J1132-5606 (TA2, TA3, TA4). Flux calibration was done using Ceres and Titan (TA1), J11315818 (TA2), J1150-5416 (TA3), and J0635-7516 and J10372934 (TA4). Based on the calibrator fluxes, we estimate the absolute flux calibration to be accurate to within $5 \%$.

The data were reduced using different versions of the Common Astronomy Software Applications package (CASA; McMullin et al. (2007)). After corrections for the time and frequency dependence of the system temperatures, and rapid atmospheric variations at each antenna using water vapour radiometer data, bandpass and gain calibration were performed. For the Band 6 setting, data obtained in three different configurations were combined. Subsequently, self-calibration was performed on the strong continuum for the Band 6 data. Imaging was carried out using the CASA tclean algorithm after a continuum subtraction was performed on the emission line data. The final line images were created using Briggs robust weighting (0.5). This resulted in beam sizes of $00^{\prime \prime} 028 \times 0^{\prime \prime} .026\left(38^{\circ}\right)$ and $0^{\prime} .22 \times 0$ !' $17\left(-48^{\circ}\right)$ for the Bands 6 and 7 data, respectively. Typical rms noises per channel are $\approx 0.6$ and $\approx 1.3 \mathrm{mJy} \mathrm{beam}^{-1}$ for the Bands 6 and 7 data at 3 and $1 \mathrm{~km} \mathrm{~s}^{-1}$ resolution, respectively.

\subsection{Archival optical spectra}

The archive of the European Southern Observatory (ESO) contains some excellent high-resolution spectra of HD 101584. These spectra were obtained with the ESO VLT/UVES instrument through the observing program 266.D-5655(A) and were retrieved by us as pipeline-processed data products. All 19 exposures were made on 2003-02-04 between 09:00 and 09:30 UT (JD 2452674.9). The spectra in the visual and red re- 


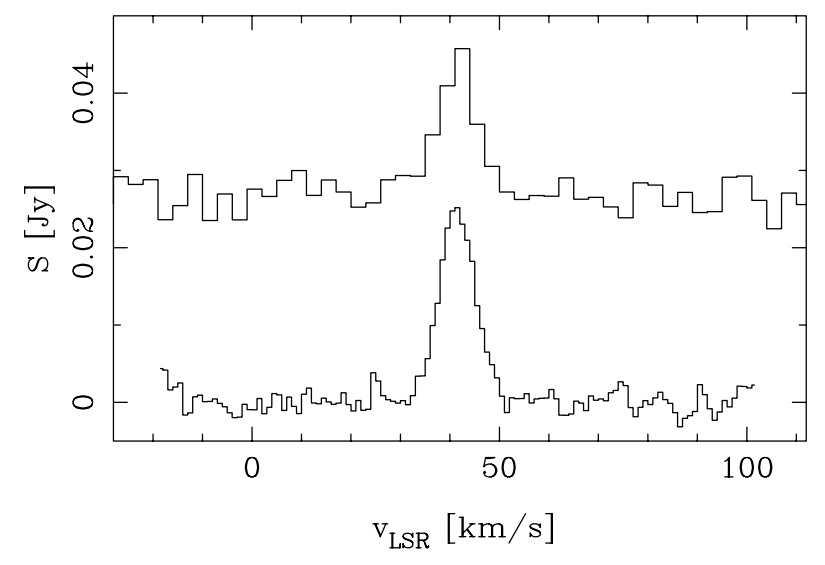

Fig. 1. X30 $\alpha$ (upper spectrum; scaled by a factor of three and biased by $25 \mathrm{mJy}, 3 \mathrm{~km} \mathrm{~s}^{-1}$ resolution) and X26 $\alpha$ (lower spectrum; $1 \mathrm{~km} \mathrm{~s}^{-1}$ resolution) lines observed towards HD 101584. Continuum emissions have been subtracted.

gion (wavelengths $4727-10426 \AA$ ) have a resolving power of $R=74450$, corresponding to a velocity resolution of $4.0 \mathrm{~km} \mathrm{~s}^{-1}$. The blue spectra (3760-4990 $\AA$ ) have $R=65030$ or a resolution of $4.6 \mathrm{~km} \mathrm{~s}^{-1}$. We performed a detailed analysis of small wavelength intervals to search for emission lines that might be associated with the region that emits the observed mm-wave emission lines.

\section{Line identification}

We detect two lines, at rest frequencies of 232.0245 and $353.8100 \mathrm{GHz}$ (adopting a systemic velocity of $41.5 \mathrm{~km} \mathrm{~s}^{-1}$ ) in our ALMA observations towards HD101584; see Fig 1. Both have Gaussian line shapes with full widths at half maximum (FWHMs) of about $9 \mathrm{~km} \mathrm{~s}^{-1}$. Both line emissions are spatially unresolved and centred on the continuum peak, which is believed to be the position of the star. The best upper limit to the lineemitting source size is obtained for the $232.0245 \mathrm{GHz}$ line, with a FWHM $\lesssim 0$ '”02 assuming a Gaussian source (after deconvolving with the beam).

The source is modestly rich in molecular lines with a line density of less than three lines per GHz (Olofsson et al. 2019), but we have found no identification in terms of relevant molecular lines ${ }^{1}$ Furthermore, the line widths differ from those of the molecular lines obtained within an aperture of about 0.2 , Sect. 6.4. On the other hand, these rest frequencies are consistent with an identification in terms of the X30 $\alpha$ and X26 $\alpha$ RTLs from an element $\mathrm{X}$ heavier than carbon. As an example, the $\mathrm{S} 30 \alpha$ and S26 $\alpha$ lines lie at 232.0233 and $353.8093 \mathrm{GHz}$. In Sect. 5] we present a simple model that makes the identification in terms of atomic RTLs viable. Nearby $\mathrm{X} n \beta$ lines lie outside the frequency coverage of our observations.

Direct identification of the carrier(s) is more difficult because the RTLs from heavy elements are close together in rest frequency, for instance, the ${ }^{23} \mathrm{Na}$ and ${ }^{56} \mathrm{Fe} 30 \alpha$ and $26 \alpha$ lines lie at 232.0217 and $232.0250 \mathrm{GHz}$ and 353.8069 and $353.8112 \mathrm{GHz}$, respectively. That is, they lie on each side of our determined rest frequencies, and well within the observed line widths that cor-

\footnotetext{
1 There is an $\mathrm{H}_{2}^{13} \mathrm{CO}\left(5_{05}-4_{04}\right)$ line at $353.8119 \mathrm{GHz}$. However, this can be ruled out as an identification because we know from previous data that the $\mathrm{H}_{2} \mathrm{CO}$ lines are strongest at the so-called extreme velocity spots (Olofsson et al. 2017 2019), and the $\mathrm{H}_{2}^{13} \mathrm{CO}\left(5_{05}-4_{04}\right)$ line is not detected in any of them.
}

respond to $\approx 7$ and $11 \mathrm{MHz}$ for the $\mathrm{X} 30 \alpha$ and $\mathrm{X} 26 \alpha$ lines, respectively. Therefore, theoretical modelling is required to make further progress.

A useful formula for calculating rest frequencies of RTLs (ignoring the fine structure of hydrogen and the electronic structure of the core for non-hydrogenic atoms), $n+\Delta n \rightarrow n$, is given by the Rydberg formula of Towle et al. (1996),

$$
\begin{aligned}
v_{n+\Delta n, n}= & 3.289842 \times 10^{6}\left(1-5.48593 \times 10^{-4} / m_{\mathrm{a}}\right) \times \\
& \times\left[\frac{1}{n^{2}}-\frac{1}{(n+\Delta n)^{2}}\right][\mathrm{GHz}],
\end{aligned}
$$

where $m_{\mathrm{a}}$ is the atomic mass of the species in atomic mass units (in the usual nomenclature $\Delta n=1$ is an $\alpha$ line, $\Delta n=2$ a $\beta$ line, etc.).

The continuum flux from the line-emitting source is difficult to estimate due to contamination with dust continuum emission. The Band 6 data have the highest resolution and using different uv-tapering we estimate flux densities of $7.5,10.1$, and $13.2 \mathrm{mJy}$ for beam sizes of about $00^{\prime \prime} 025,00^{\prime \prime} 083$, and $0 \prime$. 15 , respectively. This gives a flux density that increases only slowly with beam area, which suggests that the $7.5 \mathrm{mJy}$ within an estimated source size of 0 ".011 (the FWHM of a Gaussian source after deconvolving with the beam) is relatively free from dust emission at this frequency $\left.\right|^{2}$ The Band 7 continuum source size is 0 ". 19 , as measured by an 0 '.23 beam, and the flux density is $61 \mathrm{mJy}$. Even if the dust contribution increases only slowly with beam area, the contribution from dust continuum is likely to be significant in this case.

The line characteristics are summarised in Table 1 The line strengths are 7.3 and $25 \mathrm{mJy}$ and the line-to-free-free-continuum flux density ratios are $>1.0$ and $>0.4$ for the $30 \alpha$ and $26 \alpha$ lines, respectively. Upper limits to the corresponding $\mathrm{H}, \mathrm{He}$, and $\mathrm{C}$ lines are 1 and $2 \mathrm{mJy}$ in the two bands, respectively. That is, for the $26 \alpha$ line the $\mathrm{X} / \mathrm{H}$ line intensity ratio is $\gtrsim 10$. The lines are perfectly Gaussian, within the noise levels, and there is no evidence of low-intensity, broad line wings.

\section{A simple model in strict LTE}

The atomic lines reported here in the mm-wave spectrum of HD 101584 arise in the immediate environment of the binary system, within a few astronomical units, as shown by the observations. A complete analysis of the data requires a non-LTE model that takes into account chemistry, ionisation, excitation, and radiative transfer in a source of arbitrary geometry. However, this is far beyond the scope of this paper. Instead, we have devised a model in strict LTE that will explain the presence of heavy-element RTLs and a free-free continuum, at their observed intensities, and the simultaneous absence of the corresponding $\mathrm{C}$ and H RTLs. With such a model it is possible to construct models of the RTL emission, from an assumed geometry of the gas, with the smallest number of free parameters. The model can also be used to easily make predictions for future observations. Because of the complexity of the problem, it is difficult to argue in detail whether or not such an approach is justified, but here we present some arguments in favour of the LTE model.

In our opinion, the primary star with its modest luminosity $\left(\approx 1600[D / 1 \mathrm{kpc}]^{2} L_{\odot}\right)$ and moderate temperature $(\approx 8500 \mathrm{~K})$ is

\footnotetext{
2 The contribution to the continuum from the visible photosphere of a star with the characteristics of HD 101584 is negligible.
} 
Table 1. Observed line and continuum characteristics.

\begin{tabular}{|c|c|c|c|c|c|c|c|}
\hline Line & $\begin{array}{c}v_{\text {rest }}{ }^{a} \\
{[\mathrm{GHz}]}\end{array}$ & $\begin{array}{c}S_{\ell} \\
{[\mathrm{mJy}]}\end{array}$ & $\begin{array}{c}\Delta v^{b} \\
{\left[\mathrm{~km} \mathrm{~s}^{-1}\right]}\end{array}$ & $\begin{array}{c}\int S_{\ell} \mathrm{d} v \\
{\left[\mathrm{Jy} \mathrm{km} \mathrm{s}{ }^{-1}\right]}\end{array}$ & $\begin{array}{l}\theta_{\ell}^{c} \\
{\left[{ }^{\prime \prime}\right]}\end{array}$ & $\begin{array}{c}S_{\mathrm{c}} \\
{[\mathrm{mJy}]}\end{array}$ & $\begin{array}{c}\theta_{\mathrm{c}}{ }^{d} \\
{\left[{ }^{\prime \prime}\right]}\end{array}$ \\
\hline $\begin{array}{l}\mathrm{X} 30 \alpha \\
\mathrm{H} / \mathrm{He} / \mathrm{C} 30 \alpha\end{array}$ & $232.0245 \pm 0.0016$ & $\begin{array}{c}7.3 \pm 0.7 \\
<0.8\end{array}$ & $8.7 \pm 1.0$ & $0.074 \pm 0.007$ & $<0.02$ & $7.5 \pm 0.5$ & $0.011 \pm 0.001$ \\
\hline $\begin{array}{l}\mathrm{X} 26 \alpha \\
\mathrm{H} / \mathrm{He} / \mathrm{C} 26 \alpha\end{array}$ & $353.8100 \pm 0.00077$ & $\begin{array}{l}25.1 \pm 1.5 \\
\quad<1.2\end{array}$ & $8.5 \pm 0.2$ & $0.225 \pm 0.012$ & $<0.04$ & $61.2 \pm 3.0$ & $0.190 \pm 0.016$ \\
\hline
\end{tabular}

Notes. ${ }^{(a)}$ Assuming emission at the systemic velocity of $41.5 \pm 0.15 \mathrm{~km} \mathrm{~s}^{-1}$. $^{(b)}$ FWHM of a Gaussian fit to the line profile (deconvolved with the spectral resolution). ${ }^{(c)}$ Upper limit to the source size (FWHM of a Gaussian source after deconvolving with the beam) using a Gaussian fit to the line brightness distribution in the systemic velocity channel. ${ }^{(d)}$ Estimated source size (FWHM of a Gaussian source after deconvolving with the beam) using a Gaussian fit to the continuum brightness distribution. The $232 \mathrm{GHz}$ data are obtained with a circular beam of 0 " 025 , while those at $353 \mathrm{GHz}$ are obtained with a beam of 0 ' $24 \times 0^{\prime \prime} \cdot 17\left(19^{\circ}\right)$ and are likely to have a significant contribution from dust continuum emission.

not able to produce an ionised nebula that can explain the observed RTL and continuum flux densities and, at the same time, the absence of the corresponding C and H RTLs. First, the high density that is required to explain the observed line and continuum flux densities (see below) will lead to efficient absorption of the ionising radiation already close to the star (the density is much higher here than in the traditional nebular case). The alternative, that the RTL region is located at a distance from the star with an absorption-free cavity in between, appears less likely. Second, such a nebula, produced by a star with the characteristics of HD 101584, would produce H and C RTLs that are stronger than the corresponding heavy-element lines, in stark contrast to the observed result.

The question remains as to whether or not strict LTE is well justified. In order for the ionisation, chemical abundances, internal excitation, continuum brightness, and line intensities to be well characterised by equilibrium conditions, the rates of all microscopic processes must be in detailed balance at the same value of the temperature. The densities of the best-fitting LTE models (see below), $n_{\mathrm{H}} \approx 10^{11}-10^{12} \mathrm{~cm}^{-3}$, are higher by orders of magnitude than typical densities in the interstellar medium where non-equilibrium prevails. On the other hand, this density is still much lower than the density in a typical stellar photosphere at the same temperature, where LTE is a useful starting approximation, but where subtle non-LTE effects can nevertheless be found in ionisation and line formation of some elements. Simple estimates of chemical, photodissociation, and ionisation timescales, as well as comparisons of collisional and radiative strengths, suggest that the LTE assumption is, if not perfect, at least acceptable. In addition, the line flux density ratio, $26 \alpha / 30 \alpha=3.4 \pm 0.4$, is not too far from the blackbody ratio (in the Rayleigh-Jeans limit) for emission at 232 and $354 \mathrm{GHz}$, namely 2.3, and we therefore suspect that the lines are formed in thermal equilibrium. The slightly higher value for the observed flux density ratio than the blackbody ratio can be attributed to an optical depth effect, that is, the lines are at least partially optically thin and the optical depth of the $26 \alpha$ transition is higher than that of the $30 \alpha$ transition.

Consequently, we assume that the abundances of molecular species, the ionisation balance, and the level populations are all governed by the processes that tend to drive the gas toward the equilibrium state at the kinetic temperature prevailing in the near circumstellar environment, that is, we assume that strict local LTE applies. This assumption is also the reason behind our choice to deliberately refer to the spectroscopic features as RTLs rather than radio recombination lines throughout this paper in order to steer the reader away from any prejudice that the lines arise mainly through the process of radiative recombination or that they should behave like the well-known features in neb- ular spectra. Further, the equilibrium temperature must be low enough that ionisation of hydrogen, helium, and carbon does not occur. We compare the observational constraints (Table $1^{3}$ to a simple model of a uniform, isothermal parcel of gas in strict LTE. This parcel of gas is characterised by two parameters: a number density of hydrogen in all forms, $n_{\mathrm{H}}$, and a temperature, $T$. To this should be added the parameters defining the geometry and kinematics. Once the true geometry and kinematics of the source have been established, a more sophisticated modelling can be attempted.

\subsection{Geometry}

We have chosen a geometry in light of the VLTI/PIONIER results of Kluska et al. (2020), who infer a face-on (as seen from Earth), circumbinary dust disc with a sublimation rim at about $2[D / 1 \mathrm{kpc}]$ au from the central object. The region in which the RTLs are formed could therefore consist of dust-free gas surrounding the binary system and extending along the upper and lower surface layer of this dust disc (the estimated source size is larger than the diameter of the dust rim, see below).

Considering this, we have chosen a simple geometry in terms of a cylinder seen along its axis, where $R$ is the radius of the source in the plane of the sky and $\Delta z$ is the depth of the cylinder along the line of sight. The apparent angular diameter of the emitting region is $\theta=2 R / D$, and we specify the geometry of the emitting region by the parameter $\rho=\Delta z / R$. The case $\rho=1$ corresponds to a cylinder of radius $R$ and height $\Delta z=R$ viewed face-on at a distance $D$. When $\rho \ll 1$, the modelled flux refers to that of a very thin disc, while the $\rho=2$ case is close to that of a spheroid. We adopt $\rho=0.1$, that is, a geometrically thin disc, as the most likely geometry for describing the RTL region. This also minimises the volume of the relatively warm gas required to explain the observations.

In strict LTE, it is possible to derive a lower limit to the size of the line-emitting region. The flux densities of the lines cannot exceed that of a blackbody at a given temperature. For a temperature of $3000 \mathrm{~K}$ (it is shown below that this is a reasonable temperature in this context), both lines give about 10 mas as the lower limit to the source size. This agrees very well with the measured Gaussian size of the continuum source at $232 \mathrm{GHz}$, namely 11 mas. This is our best estimate for the source size because the $232 \mathrm{GHz}$ data have a much higher angular resolution than those at $354 \mathrm{GHz}$, and the continuum data have a much

\footnotetext{
3 The observations of the two lines were made at different times; therefore, it is possible that variability has affected the ratio of their fluxes. Even so, we treat the measurements as though they were contemporaneous and assume that both lines arise in the same emitting gas.
} 
higher $\mathrm{S} / \mathrm{N}(\approx 70)$ than the line data $(\approx 13)$. We make the reasonable assumption that the line and continuum emissions come from the same region. As shown by van Hoof (2000) the relation between the true source size and the size of a Gaussian fit to its brightness distribution depends on the geometry of the source; for instance, a uniform brightness disc is about 1.5 times larger than the FWHM of the Gaussian fit to its brightness distribution. We will nevertheless adopt 11 mas as the size of the RTL region, that is, the assumed thin disc extends to $R=5.5[D / 1 \mathrm{kpc}]$ au. The reason is twofold: first, and most importantly, the resulting density and temperature depend only weakly on the source size (see Sect. 6.2.3 and Appendix D, and secondly, in our case the true source geometry is unknown.

Of course, other geometries are not excluded, keeping in mind that this is at the heart of the complex circumstellar morphology as shown by our ALMA data (Olofsson et al. 2019). In all likelihood, an accretion disc, an associated jet, and the innermost part of an hourglass structure are located in the region (Olofsson et al. 2019). However, as shown in Appendix D, results such as mass, density, and temperature of the gas depend little on the assumed geometry.

\subsection{Chemistry}

We consider an ideal gas composed of hydrogen and the 14 other most abundant elements of comparable or smaller first ionisation potential. Helium is included, although it remains fully neutral at the temperatures of interest here. Two different sets of abundances are compared in the analysis, as summarised in Table A.1. The reference abundances are those of the solar photosphere $(Y$; Set 1$)$, and Set 2 consists of abundances depleted by a factor of $d$.

In addition to the elemental composition we take into account the formation of some simpler molecules, as this may have important consequences for the atomic abundances, the atomic ionisation balance, and for the dominant sources of continuous opacity in the gas, which depend on collisions between free electrons and ions and the principal neutral species $\mathrm{H}, \mathrm{H}_{2}$, and $\mathrm{He}$. We therefore calculated the molecular abundances (at each grid point) for the species given in Table B.1 ${ }^{4}$ We used the most recent values for partition functions and dissociation energies in the calculation of the equilibrium abundances. The molecular partition function is a sum over all low-lying electronic states and enough of their vibrational and rotational levels to yield a converged value at temperatures of interest. The full list of included molecules, their dissociation energies, and abundances in the reference model is presented in Table B.1 of Appendix B.

It should be noted here that recombination lines from molecules can be ignored because the recombination of a molecular ion and an electron preferentially leads to a dissociation of the molecule.

\subsection{Ionisation balance}

All elements $\mathrm{X}_{i}$ are taken to exist in two ionisation states, the neutral atom $\mathrm{X}_{i}^{0}$ and first ion $\mathrm{X}_{i}^{+1}$, except hydrogen, which also forms a potentially important anion, $\mathrm{H}^{-}$. Each internal energy state in the gas is labelled with the indices $i$ for the element, $j=0, \pm 1$ for the charge state, and $k$ for the quantum state of energy $E_{i j k}$. In strict LTE, the population of state $i j k, p_{i j k}$, can be expressed as a number density given by the Boltzmann formula,

\footnotetext{
4 In the chemistry calculations, the elements $\mathrm{N}$ and $\mathrm{O}$ also play important roles. They are assumed to take on their solar abundances.
}

$$
\frac{p_{i j k}}{p_{i j}}=\frac{g_{i j k} \exp \left(-E_{i j k} / k_{\mathrm{B}} T\right)}{Q_{i j}(T)}
$$

where

$Q_{i j}(T)=\sum_{k} g_{i j k} \exp \left(-E_{i j k} / k_{\mathrm{B}} T\right)$

is the partition function and $p_{i j}$ the total density of the ion $\mathrm{X}_{i}^{j}$ summed over all its states, $p_{i j}=\sum_{k} p_{i j k}$. For each element $i$, the sum over all states of all its ions is related to the total number density of hydrogen in all forms, $n_{\mathrm{H}}$, by

$\sum_{j} p_{i j} \equiv \sum_{j} \sum_{k} p_{i j k}=n_{\mathrm{H}} 10^{\left(Y_{i}-Y_{\mathrm{H}}\right)}$.

The ionisation balance is governed by the Saha equation

$\frac{p_{i 1} p_{e}}{p_{i 0}}=\frac{Q_{i 1} Q_{e}}{Q_{i 0}} \exp \left(-I_{i} / k_{\mathrm{B}} T\right)$,

where

$Q_{e}=\left(\frac{2 \pi m_{e} k_{\mathrm{B}} T}{h^{2}}\right)^{3 / 2}$

is the partition function of the continuum of free-electron states (translational energies), $I_{i}$ is the first ionisation potential of element $i, k_{\mathrm{B}}$ is Boltzmann's constant, $h$ is Planck's constant, and $m_{\mathrm{e}}$ is the electron mass. Overall charge neutrality is assumed, so that the density of free electrons is given by

$p_{e}=\sum_{i} p_{i 1}-p_{\mathrm{H},-1}$.

Given a set of element abundances $Y_{i}$ and the two parameters $T$ and $n_{\mathrm{H}}$, Eqs. (2) to (7) are solved simultaneously by iteration. The result is a complete set of densities, $p_{i j k}$. We computed the partition functions of neutral atoms and first ions by direct summation over tables of energy levels derived from the NIST Atomic Spectra Database 5 .

\subsection{Radiative transfer}

Once the populations have been determined, the continuum opacity can be evaluated. At radio, mm, submm, and far-infrared wavelengths, the important processes in the weakly ionised gas are electron-ion bremsstrahlung (free-free), electron-neutral bremsstrahlung, and Thomson scattering. The electron-ion freefree absorption coefficients are computed using the accurate Gaunt factors of van Hoof et al. (2014). We compute the electron-neutral $\left(\mathrm{e}^{-}+\mathrm{H}\right)$ free-free absorption coefficients based on the analytical approximation of Stallcop (1974), which agrees within 5\% with accurate calculations (Bell \& Berrington 1987, John 1994d) at wavelengths $\lambda>1 \mu \mathrm{m}$ and temperatures $T=1400$ to $10080 \mathrm{~K}$. We also include $e^{-}+\mathrm{He}$ (John 1994a b) and $e^{-}+\mathrm{H}_{2}$ (John 1994c) in the electron-neutral free-free opacity. In principle, electrons on polar, neutral molecules like $\mathrm{CO}$ and $\mathrm{H}_{2} \mathrm{O}$ produce very large free-free opacities per molecule (John 1975b a ); however, they contribute relatively little to the total compared 5 The U.S. National Institute of Standards and Technology (NIST)
maintains a critically reviewed database of atomic energy levels at
https://physics.nist.gov/PhysRefData/ASD/

levels_form.html 
with the more abundant $\mathrm{H}, \mathrm{H}_{2}$, and $\mathrm{He}$ in our models. In order to describe the opacity at mid-infrared and shorter wavelengths $(\lambda<50 \mu \mathrm{m})$, we include non-relativistic Thomson scattering by free electrons and $\mathrm{H}^{-}$bound-free absorption (crosssections from McLaughlin et al. (2017ba)).

These absorption coefficients are conventionally expressed as an inverse length per unit density of the atom or ion and per unit electron pressure, $\kappa$, where the electron pressure is $P_{\mathrm{e}}=p_{\mathrm{e}} k_{\mathrm{B}} T$. The optical depth in the continuum through a uniform medium of length $\Delta z$ is thus

$\tau_{\mathrm{c}}=P_{\mathrm{e}}\left(p_{\mathrm{H}} \kappa_{\mathrm{e}, \text { neutral }}+p_{\mathrm{e}} \kappa_{\mathrm{e}, \text { ion }}\right) \Delta z$.

Under the assumption of a Gaussian line profile with FWHM $\Delta v$, the corresponding optical depth at the centre of a line of element $i$ in ion stage $j$ is given by

$\tau_{n^{\prime} n^{\prime \prime}}=3.738 \times 10^{-7} A_{n^{\prime} n^{\prime \prime}} p_{i j n^{\prime \prime}}\left(\frac{g_{n^{\prime}}\left[1-\exp \left(-h v / k_{\mathrm{B}} T\right)\right]}{g_{n^{\prime \prime}}(v / c)^{3} \Delta v}\right) \Delta z$,

where $g_{n^{\prime}}$ and $g_{n^{\prime \prime}}$ are the statistical weights of the respective upper and lower states of the transition $n^{\prime} \rightarrow n^{\prime \prime}, A_{n^{\prime} n^{\prime \prime}}$ is the spontaneous transition probability $\left[\mathrm{s}^{-1}\right], c$ is the speed of light $\left[\mathrm{cm} \mathrm{s}^{-1}\right]$, and the line width has dimensions $\left[\mathrm{km} \mathrm{s}^{-1}\right]$. The product $p_{i j n^{\prime \prime}} \Delta z$ is the lower-state column density with dimensions $\left[\mathrm{cm}^{-2}\right]$. With the assumption of LTE, the excitation temperature of any transition is equal to $T$, the source function is equal to the Planck function $B_{v}(T)$, and the observable flux density of either the line peak or continuum is

$S_{v}=B_{v}(T)[1-\exp (-\tau)] \pi\left(\frac{R}{D}\right)^{2}$

that is, the line and continuum emissions are treated separately. This requires, for instance, optically thin emission, a situation that applies reasonably well to our models. To summarise the model, Eqs. (2) through (10) show how the observable flux densities of line and continuum depend on the parameters $n_{\mathrm{H}}, T$, $\theta$ (or $R$ ), $\rho$, and $D$ for a specified set of elemental abundances, where $n_{\mathrm{H}}=n_{\mathrm{H}^{0}}+n_{\mathrm{H}^{+}}+n_{\mathrm{H}^{-}}+2\left(n_{\mathrm{H}_{2}}+n_{\mathrm{H}_{2}^{+}}\right)+3 n_{\mathrm{H}_{3}^{+}}$.

\subsection{Line broadening: Doppler motions and blending of emitters}

The shapes and widths of the line profiles contain valuable information. The observed lines are fit very well by single Gaussian functions, with a FWHM of $\Delta v \approx 8.5 \mathrm{~km} \mathrm{~s}^{-1}$ (taken from the $\mathrm{X} 26 \alpha$ line that is observed with the highest spectral resolution). It is significant that the $26 \alpha$ and $30 \alpha$ lines show the same value of line width, and that this line width is substantially larger than the thermal line widths at the relevant temperatures (e.g. for neutral $\mathrm{Mg}$ it is only $2.4 \mathrm{~km} \mathrm{~s}^{-1}$ at $T=3000 \mathrm{~K}$ ).

Typically this would be explained by a combination of thermal Doppler broadening and micro-turbulence, with only minor contributions from any larger scale kinematical motions. For simplicity, we adopt this description in the determination of the best-fit equilibrium model, the reference model, and separate the two components into thermal Doppler motions $\left(\Delta v_{\text {th }}\right)$ and micro-turbulent motions $\left(\Delta v_{\text {turb }}\right)$. We further assume that both have Gaussian broadening functions along the line of sight through the emitting region. The Gaussian widths add in quadrature $\Delta v^{2}=\Delta v_{\text {th }}^{2}+\Delta v_{\text {turb }}^{2}$.

Broadening of the lines results also from the fact that each line is most likely a blend of lines due to a mixture of different species. The slight differences in reduced mass of this species lead to slight differences in line frequencies; see Eq. (1). These velocity differences are independent of $n$ for any $\alpha$ transition.

In the modelling, we obtained solutions by linear combination of optical depths of overlapping lines of the different atoms in the Rydberg approximation. The best-fitting models have line optical depths that are less than or of the order of unity, which justifies simply summing the optical depth at each point on a densely sampled frequency grid. In the construction of a theoretical profile, each of the line components is weighted by the relative abundance of the atomic species.

Rydberg atoms are very sensitive to perturbations by collisions with other atoms and electrons and by magnetic forces. Line profiles broadened by such perturbations provide limits on gas density and magnetic field strength as discussed in Appendix Cand Sect. 6.2.2 below.

\section{Results from modelling mm-wave RTLs}

The model described in the preceding section can be used to gain insight into the conditions that make mm-wave RTLs observable towards stars that are only moderately warm. We have several observable properties and a model with four free parameters. We start by explaining the adopted procedure for finding the best-fit model.

\subsection{Procedure for selecting the best-fit model}

To find the model that best fits the observations is not trivial, and therefore we describe the procedure employed:

- Adopt a distance $D$ and a set of elemental abundances.

- Select a value of the geometrical parameter $\rho$.

- For one line, specify the peak flux density $S_{v}$ and observed line width $\Delta v$.

- Specify a grid of values of the parameters $n_{\mathrm{H}}$ and $T$.

- For each set of density and temperature, compute the peak line flux densities for a list of lines, recalling that the observed line might be an unresolved blend of several emitters.

- Iterate over values of the source radius $R$ until the model matches the line flux.

- Examine the resulting grid of solutions to see which of them also satisfies the upper limit on the hydrogen line flux, the flux of the adjacent continuum, and the observed constraints on angular size of the source.

- For the same choice of $D$, abundances, and $\rho$, repeat the above steps for a second transition.

- Determine whether there is some parameter space that satisfies both transitions and their continuum fluxes simultaneously.

- Repeat the entire procedure for different choices of elemental abundances and $\rho$.

The line list for the RTLs includes two lines ( $\mathrm{H}$ and $\mathrm{C}$ ) and one blend (all abundant heavier elements that contribute to the blend, $\mathrm{Na}$ to $\mathrm{Ni}$ ). The densities of the neutral species have been computed explicitly in strict LTE. The mathematical model is non-linear in several regards. The temperature enters in the arguments of exponential functions in the Saha and Boltzmann equations describing the ionisation balance and excitation. The source path length $\Delta z=\rho R$ is a term in each optical depth and thus enters in the arguments of exponential functions. The coupled parameter for the radius enters as $R^{2}$ in the flux densities. Thus, the solutions must be found by successive iterations. Remarkably, in the present case, the solutions appear to converge on a relatively small volume in parameter space. 

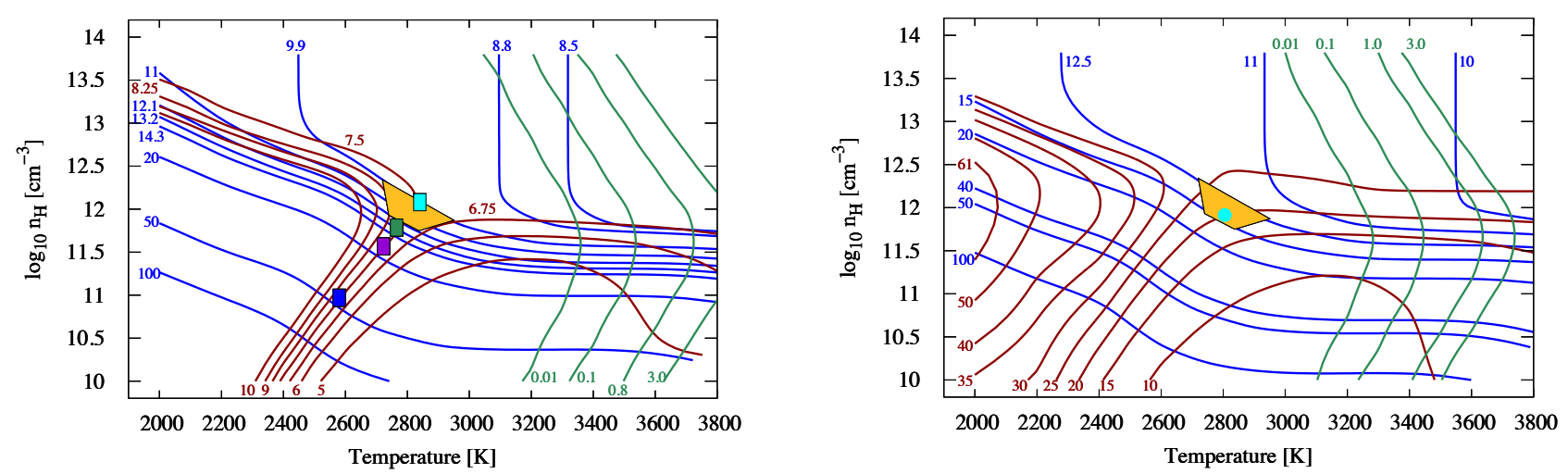

Fig. 2. Left: LTE solutions with $\rho=0.1$ that yield a line flux density of $7.3 \mathrm{mJy}$ in the $30 \alpha$ blend for solar abundances. Blue contours display equal values of the angular diameter $\theta$ in mas. Dark red contours show the $232 \mathrm{GHz}$ continuum flux density in mJy. Green contours show the flux density of the corresponding hydrogen line in mJy. The polygon covers the space of primary solutions that allow for $\pm 10 \%$ changes on the continuum flux density and the angular diameter for illustrative purposes. The shaded rectangles indicate how the primary solution shifts when the value of $\rho$ is changed by a factor of two (0.05 (violet) and 0.20 (green)), and when a source size of 17 mas and $\rho=2$ are adopted (blue). Right: Same as left panel but for the $26 \alpha$ blend and a line flux density of $25 \mathrm{mJy}$, and the $354 \mathrm{GHz}$ continuum flux density in mJy. The cyan solid circle indicates the location of the reference model.

\subsection{The reference model}

The behaviour of the models is illustrated in two contour diagrams shown in Fig. 22 These diagrams of the $\left(\log n_{\mathrm{H}}, T\right)$-plane display contours of equal angular diameter ( $\theta$ in mas, blue), continuum flux density $\left(f_{\mathrm{c}}\right.$ in mJy, brown), and hydrogen line flux ( $f_{\mathrm{H}}$ in $\mathrm{mJy}$, green). These models were computed for $\rho=0.1$ (a cylinder with a depth equal to a tenth of its radius), undepleted solar abundances, a nominal distance of $D=1.0 \mathrm{kpc}$, and the line flux densities given in Table 1 that is, all points in the diagrams give the correct observed line intensities. However, the observational results will limit the area of acceptable solutions, for instance, the upper limits on the $\mathrm{H} 30 \alpha$ and $\mathrm{H} 26 \alpha$ lines imply that the temperature must be $T \lesssim 4000 \mathrm{~K}$, for the adopted solar abundances, and the measured source size is about 11 mas.

The $\mathrm{X} 30 \alpha$ transition provides the most restrictive constraints, because of the higher angular resolution of the observations. As shown in Fig. 2. left, there are two regions of the $\left(n_{\mathrm{H}}, T\right)$ plane that satisfy all of the observed constraints of the $30 \alpha$ transition and adjacent continuum. We pick the midpoint of the polygonic area in the left panel of Fig. 2 as the reference model. The physical characteristics of this region are $T=2800 \mathrm{~K}$ and $n_{\mathrm{H}}=8 \times 10^{11} \mathrm{~cm}^{-3}$, and its size is $R=5.5 \mathrm{au}$. The resulting gas mass is $\approx 7 \times 10^{-4} M_{\odot}$, that is, only about $2 \%$ of the estimated mass of the CCS component. The ionisation fraction is low, $\approx 3 \times 10^{-5}$, and $\mathrm{CO}$ dominates over $\mathrm{H}_{2}$ with $[\mathrm{CO}] /\left[\mathrm{H}_{2}\right] \approx 6$ and $[\mathrm{CO}] /[\mathrm{H}] \approx 3 \times 10^{-4}$ (a significant fraction of the $\mathrm{C}$ is bound in $\mathrm{CO}$ ). The corresponding $\mathrm{H}$ and $\mathrm{C}$ recombination lines are more than a million times weaker than the heavy-element blend. The properties of the reference model are given in Tables 2 and 3 . The corresponding solutions for the $26 \alpha$ transition are displayed in the right panel of Fig. 2. Remarkably, the solutions for this transition cluster in nearly the same part of parameter space as for the $30 \alpha$ transition.

The contours of the $232 \mathrm{GHz}$ continuum flux density do not close in the upper part of the diagram. As a result, there is a secondary solution at higher density $\left(2.2 \times 10^{13} \mathrm{~cm}^{-3}\right)$ and lower temperature $(2050 \mathrm{~K})$. However, this solution is implausible for two reasons. A very large continuum optical depth is required in order for this model to produce the observed line flux within the observed angular size. This would imply that the $\tau_{\mathrm{c}} \sim 1$ surface of the continuum source is much more extended than the line emission, in contradiction to the $232 \mathrm{GHz}$ line and continuum
Table 2. Properties of the reference model defined by $T=2805 \mathrm{~K}$, $n_{\mathrm{H}}=8.2 \times 10^{11} \mathrm{~cm}^{-3}, R=5.5 \mathrm{au}$, and $M=7.2 \times 10^{-4} M_{\odot}(\rho=0.1)$.

\begin{tabular}{lc}
\hline \hline Flux densities & {$[\mathrm{mJy}]$} \\
\hline$S_{v}(\mathrm{X} 30 \alpha)$ & 7.3 \\
$S_{v}(\mathrm{H} 30 \alpha)$ & $2.2 \times 10^{-6}$ \\
$S_{v}(\mathrm{C} 30 \alpha)$ & $2.0 \times 10^{-8}$ \\
$S_{\mathrm{c}}(232 \mathrm{GHz})$ & 7.5 \\
$S_{v}(\mathrm{X} 26 \alpha)$ & 25 \\
$S_{v}(\mathrm{H} 26 \alpha)$ & $7.0 \times 10^{-6}$ \\
$S_{v}(\mathrm{C} 26 \alpha)$ & $6.5 \times 10^{-8}$ \\
$S_{\mathrm{c}}(354 \mathrm{GHz})$ & 20 \\
\hline \hline Optical depths & \\
\hline$\tau_{\ell}(232 \mathrm{GHz})$ & 1.2 \\
$\tau_{\mathrm{c}}(232 \mathrm{GHz})$ & 1.3 \\
$\tau_{\ell}(354 \mathrm{GHz})$ & 1.1 \\
$\tau_{\mathrm{c}}(354 \mathrm{GHz})$ & 0.7 \\
\hline \hline $\mathrm{Contribution}$ to the X26 $\alpha$ flux density & \\
\hline Element & {$[\%]$} \\
\hline $\mathrm{Na}$ & 6.6 \\
$\mathrm{Mg}$ & 74 \\
$\mathrm{Al}$ & 11 \\
$\mathrm{Si}$ & 1.9 \\
$\mathrm{Ca}$ & 8.3 \\
$\mathrm{Fe}$ & 1.9 \\
\hline
\end{tabular}

maps. Secondly, the secondary solution implies much higher molecular abundances than the primary model because it is both denser and colder. The result is that the predicted LTE emission line fluxes of lines of $\mathrm{CO}$ and $\mathrm{SiO}$ in the observed bands are much larger than those observed; see Sect. 6.4 .

There are no reasonable solutions for the $\mathrm{X} 30 \alpha$ line with depleted interstellar abundances. Depleted abundances of the heavier elements (especially $\mathrm{Mg}, \mathrm{Si}$, and $\mathrm{Fe}$ ) cause the corresponding hydrogen line to be stronger, relative to the lines of the heavier elements, than in the undepleted case. The fluxes of $\mathrm{H} 30 \alpha$ and/or the $232 \mathrm{GHz}$ continuum are too high over the entire parameter space explored ( $T=1500$ to $10000 \mathrm{~K})$. 

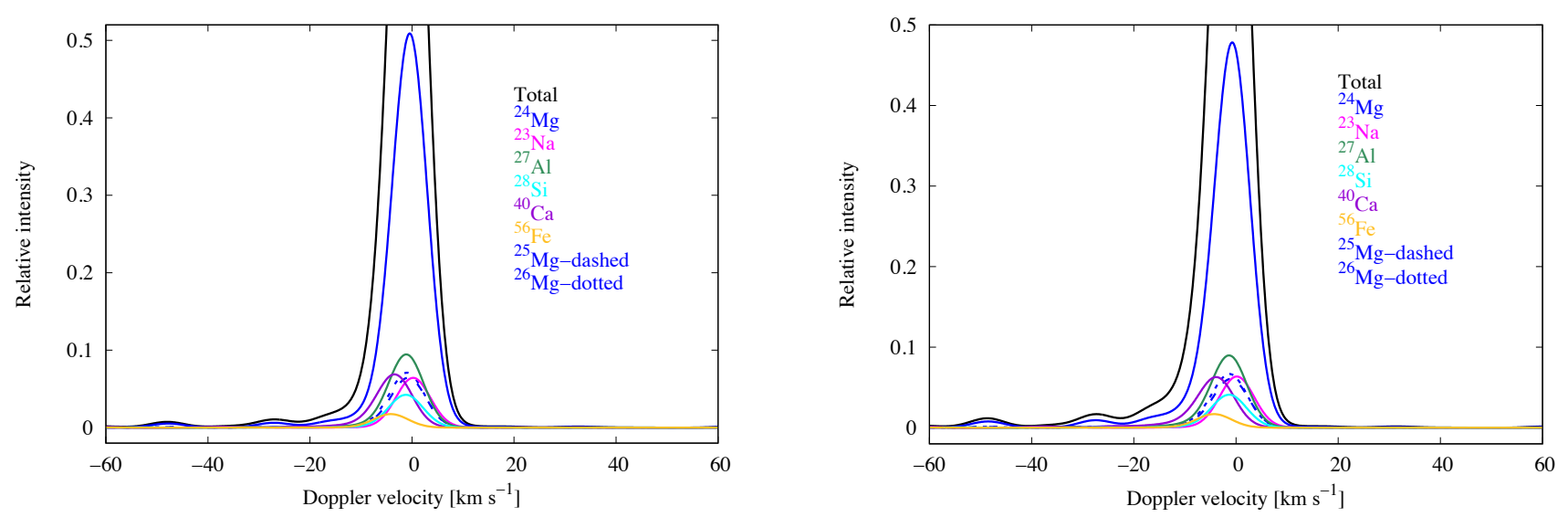

Fig. 3. Computed model profiles of the $30 \alpha$ (left) and $26 \alpha$ (right) transitions, excluding collisional broadening and radiative damping, are shown in black (truncated at a value of 0.5 , the peak value is 1.0 ). The blended profiles of the eight atoms and isotopes are shown in colours to illustrate their relative contributions and the mass-shifts in frequency. All of the profiles are slightly asymmetrical owing to the fine structure as described in Appendix C A turbulent velocity $\Delta v_{\text {turb }}=7.5 \mathrm{~km} \mathrm{~s}^{-1}$ is adopted so that the fitted FWHMs of the model profiles match the observed width, $8.5 \mathrm{~km} \mathrm{~s}^{-1}$.
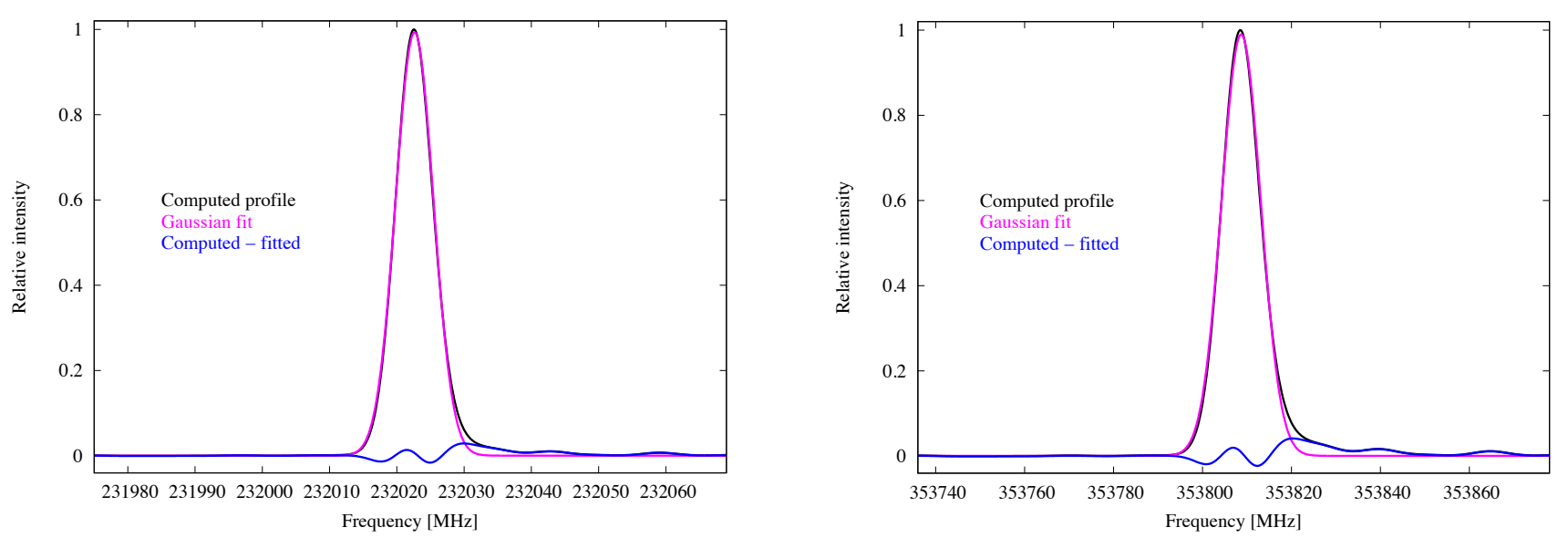

Fig. 4. Computed model profiles of the $30 \alpha$ (left) and $26 \alpha$ (right) transitions in Fig. 3 are shown in black, together with the best-fitting single Gaussians in cyan. The blue solid line is the difference between them.

\subsubsection{Identification of the line emitters}

Our LTE models further indicate that the observed lines are blends of the corresponding RTLs of $\mathrm{Na}, \mathrm{Mg}$ (including its three stable isotopes), $\mathrm{Al}$, and $\mathrm{Ca}$, with smaller contributions from $\mathrm{Si}$ and $\mathrm{Fe}$ if these elements are present in the gas at nearly solar abundances (even though Si has a greater abundance than any of these elements, its contribution to the line emission in strict LTE is smaller, because its ionisation potential is significantly higher); see Table 2. $\mathrm{Mg}$ is the largest contributor, providing $74 \%$ of the total intensity.

We computed theoretical line profiles for the $26 \alpha$ and $30 \alpha$ transitions for the blend of these eight atomic species (including isotopic variants) in the reference model. The computed model profile represents the weighted sum of the line profiles of the species. Each line component is broadened by a Gaussian of total Doppler width $\Delta v$. The thermal width is calculated separately for each atom, because it depends upon the atomic mass, while a single value of the micro-turbulent width was assumed for all $\left(\Delta v_{\text {turb }}=7.5 \mathrm{~km} \mathrm{~s}^{-1}\right.$ is adopted so that the fitted FWHMs of the model profiles match the observed width, $8.5 \mathrm{~km} \mathrm{~s}^{-1}$ ). The computed profiles are displayed in Fig. 3. The profile shapes of the two transitions are very similar, although the $30 \alpha$ profile is slightly narrower in velocity units than the $26 \alpha$ profile (zero velocity corresponds to the rest frequency of ${ }^{24} \mathrm{Mg}$ in the simple
Rydberg formula). The small shift of the blended profile relative to $\mathrm{Mg}$ is quantified in Fig. 4 through a least-squares fit of single Gaussian functions to the computed profiles. The fits determine a central rest frequency for the model blends, which are $232022.635 \pm 0.002 \mathrm{MHz}$ and $353808.691 \pm 0.004 \mathrm{MHz}$ for the $30 \alpha$ and $26 \alpha$ transitions, respectively. These values agree very well with the rest frequencies of the observed lines estimated assuming a systemic velocity of $41.5 \mathrm{~km} \mathrm{~s}^{-1}$; see Table 1 . The fits also show that the deviations from a Gaussian shape are small, less than 3.0 and $4.2 \%$ for the $30 \alpha$ and $26 \alpha$ transitions, respectively.

\subsubsection{Line broadening}

As outlined in Appendix C we can expect line broadening due to fine structure, collisional broadening, radiative damping, and Zeeman splitting in the presence of an external magnetic field, in addition to that of Doppler shifts due to thermal and turbulent motions.

Unfortunately, there is some uncertainty about the collisional broadening coefficients: adopting the nominal values, the total damping (collisional plus radiative) corresponds to a Lorentzian line shape with FWHMs of $\Gamma=2.63$ and $3.28 \mathrm{MHz}$ for $26 \alpha$ and $30 \alpha$, respectively, in the reference model. With this choice, the 
Table 3. Ionisation balance and chemical abundances (by number) in the reference model defined by $T=2805 \mathrm{~K}, n_{\mathrm{H}}=8.2 \times 10^{11} \mathrm{~cm}^{-3}$, $R=5.5 \mathrm{au}$, and $M=7.2 \times 10^{-4} M_{\odot}(\rho=0.1)$.

\begin{tabular}{lllll}
\hline \hline Element & Neutral & First Ion & \multicolumn{2}{l}{ Molecule (leading) } \\
\hline $\mathrm{H}$ & 1.0 & $4.8 \times 10^{-12}$ & $\mathrm{H}_{2}$ & $4.6 \times 10^{-5}$ \\
$\mathrm{He}$ & $8.3 \times 10^{-2}$ & $2.8 \times 10^{-32}$ & & \\
$\mathrm{C}$ & $4.4 \times 10^{-7}$ & $4.4 \times 10^{-14}$ & $\mathrm{CO}$ & $2.7 \times 10^{-4}$ \\
$\mathrm{~N}$ & $2.2 \times 10^{-5}$ & $9.3 \times 10^{-18}$ & $\mathrm{~N}_{2}$ & $2.3 \times 10^{-5}$ \\
$\mathrm{O}$ & $2.2 \times 10^{-4}$ & $8.9 \times 10^{-16}$ & $\mathrm{CO}$ & $2.7 \times 10^{-4}$ \\
$\mathrm{Na}$ & $2.3 \times 10^{-10}$ & $1.7 \times 10^{-6}$ & & \\
$\mathrm{Mg}$ & $2.0 \times 10^{-5}$ & $1.9 \times 10^{-5}$ & & \\
$\mathrm{Al}$ & $3.7 \times 10^{-8}$ & $2.8 \times 10^{-6}$ & & \\
$\mathrm{Si}$ & $3.1 \times 10^{-5}$ & $1.2 \times 10^{-6}$ & $\mathrm{SiO}$ & $3.4 \times 10^{-7}$ \\
$\mathrm{P}$ & $2.6 \times 10^{-7}$ & $2.2 \times 10^{-12}$ & & \\
$\mathrm{~S}$ & $1.3 \times 10^{-5}$ & $2.0 \times 10^{-14}$ & & \\
$\mathrm{Cl}$ & $3.2 \times 10^{-7}$ & $6.2 \times 10^{-17}$ & & \\
$\mathrm{~K}$ & $5.2 \times 10^{-13}$ & $1.1 \times 10^{-7}$ & & \\
$\mathrm{Ca}$ & $4.1 \times 10^{-9}$ & $2.2 \times 10^{-6}$ & & \\
$\mathrm{Ti}$ & $2.9 \times 10^{-9}$ & $8.6 \times 10^{-8}$ & & \\
$\mathrm{Fe}$ & $2.5 \times 10^{-5}$ & $6.4 \times 10^{-6}$ & & \\
$\mathrm{Ni}$ & $1.6 \times 10^{-6}$ & $3.7 \times 10^{-8}$ & & \\
\hline $\mathrm{e}^{-}$ & $3.4 \times 10^{-5}$ & & & \\
$\mathrm{H}^{-}$ & $4.4 \times 10^{-13}$ & & & \\
\hline
\end{tabular}

convolved profile has a Doppler core that can be fit rather well by a Gaussian of $\Delta v=8.5 \mathrm{~km} \mathrm{~s}^{-1}$, as in the observed profile, provided that the turbulent width is $\Delta v_{\text {turb }}=5.5 \mathrm{~km} \mathrm{~s}^{-1}$. The deviation from a Gaussian profile is modest, reaching at most $7 \%$ of the peak intensity in the blue wing. According to the present theory, the electron-impact collisional broadening has a strong $n$-dependence at higher values of $n$. If this applies also to lower values of $n$, the $30 \alpha$ line is expected to be markedly broader than the $26 \alpha$ line if the density is high enough. This can be used to estimate an upper limit to the density because the observed lines are well fitted with Gaussians of the same width and no indications of Lorentzian wings. Using this (see Appendix C) it appears that we rule out densities higher than $n_{\mathrm{H}} \gtrsim 3 \times 10^{12} \mathrm{~cm}^{-3}$, a result independent of the excitation models.

Finally, models with Zeeman splitting have been computed assuming a uniform magnetic field throughout the RTL region. A strong upper limit to the magnetic field of $2 \mathrm{G}$ can be set because otherwise the $30 \alpha$ line would have a larger line width than the observed one even in the absence of turbulence and damping (and would show a noticeable Zeeman triplet structure). Allowing a combination of turbulence and damping that smooths out the structure of the Zeeman triplet decreases the upper limit on the magnetic field to about $1 \mathrm{G}$.

\subsubsection{Dependence on geometry and distance}

An analysis of the sensitivity of the model results to the adopted geometry and distance is presented in Appendix D and summarised in Tables D.1 and D.3. The primary model solution is not very sensitive to the thickness of the cylinder. As an example of the dependence on geometry, the primary models for $\rho$ between 0.05 and 0.20 are shown in the contour diagram (left panel of Fig. 2), and they both fall within the hexagon of preferred solutions for $\rho=0.1$. Even for $\rho=2$, the temperature and density of the primary solution are not substantially different, $T=2650 \mathrm{~K}, n_{\mathrm{H}}=2 \times 10^{11} \mathrm{~cm}^{-3}$, and are certainly well within the uncertainties of our adopted model.
The primary model is not very sensitive to the source size either. If we adopt a source size of 17 mas, that is, the proper size of a uniform brightness disc for which the Gaussian size is 11 mas, and $\rho=0.1$, the resulting temperature and density, $2725 \mathrm{~K}$ and $4 \times 10^{11} \mathrm{~cm}^{-3}$, are only marginally different from those of the reference model, and are well within the uncertainties of our adopted model. The effect of changing both the source size ( 17 mas) and the geometry $(\rho=2)$ is illustrated by the blue rectangle in the left panel of Fig. 2. We note that a larger source results in lower optical depths for both lines and continuum.

Similarly, we recomputed the models for different values of the distance, $D=0.5$ and $2 \mathrm{kpc}$. The primary solutions are very similar to those for the adopted distance of $1 \mathrm{kpc}$ because the radius $R$ simply scales to match the observed angular size. Only the gaseous mass depends significantly on the distance. The primary solutions for $\rho=0.1$ have gas masses of $1.2 \times 10^{-4}$ and $4.2 \times 10^{-3} M_{\odot}$ for the closer and more distant alternatives, respectively, that is, the gas mass scales roughly as $D^{2.5}$.

\subsection{Constraints from optical emission lines}

HD 101584 has an optical spectrum that is rich in emission and absorption lines. Bakker et al. (1996) made a thorough analysis of their spectra obtained with the ESO Coude Auxiliary Telescope, but we note here that they assumed an edge-on geometry as opposed to the face-on geometry advocated by Olofsson et al. (2019) and Kluska et al. (2020). Here we only select a few emission lines of low optical depth in order to perform a sanity check of the model derived from the radio data.

Numerous emission lines in the visible spectrum can be identified as low-excitation transitions in neutral atoms and a few singly ionised ions. While many of these lines may arise in the chromosphere and inner wind of the star, it is possible that some lines originate in the more extended RTL region that is responsible for the mm-wave line emission. We focused on lines that are most likely to be optically thin, and extracted VLT/UVES data from the ESO archive; see Sect. 3.2. A selection of such lines are listed in Table E.1. The amplitude, centre, and width of each line was determined from a simultaneous least-squares fit of a low-order polynomial continuum and several Gaussian functions to a small wavelength interval, typically $50 \AA$. The radial velocities, near $41.5 \mathrm{~km} \mathrm{~s}^{-1}$ with respect to the LSR, are in excellent agreement with the systemic velocity of the star and with the derived radial velocities of the X26 $\alpha$ and X $30 \alpha$ lines at $\mathrm{mm}$ wavelengths. However, the observed line widths are significantly larger than those of the $\mathrm{mm}$ wave lines, in the range of 15 to $20 \mathrm{~km} \mathrm{~s}^{-1}$. These types of lines were also discussed by Bakker et al. (1996).

It is interesting to consider whether the RTL-region contributes to any of the observed emission lines in the visible spectrum. Although a full analysis of the visible spectrum is beyond the scope of this paper, a few quantitative comments can be made about. We tried to ensure that the description of the principal continuum opacities applies both in the long-wavelength limit at $\mathrm{mm}$ wavelengths and throughout the visible spectrum. At visible wavelengths, both $\mathrm{H}^{-}$bound-free opacity and Thomson scattering become important compared with the bremsstrahlung that dominates at $\mathrm{mm}$ wavelengths. It is re-assuring that the reference model of the RTLs is not in conflict with the intensities of the visible emission lines; see Appendix E. 
Table 4. Predicted radio line flux densities in the reference model ${ }^{a}$

\begin{tabular}{lllc}
\hline \hline Species & Transition & $\begin{array}{l}\text { Frequency } \\
{[\mathrm{GHz}]}\end{array}$ & $\begin{array}{c}S_{v} \\
{[\mathrm{mJy}]}\end{array}$ \\
\hline $\mathrm{Mg}$ I & $38 \alpha$ & 115.3 & 2.1 \\
$\mathrm{Mg}$ I & $54 \gamma$ & 115.6 & 0.5 \\
$\mathrm{Mg} \mathrm{I}$ & $30 \alpha$ & 232.0 & 5.9 \\
$\mathrm{Mg} \mathrm{I}$ & $26 \alpha$ & 353.8 & 10 \\
$\mathrm{Mg}$ I & $21 \alpha$ & 662.7 & 23 \\
$\mathrm{C} \mathrm{I}$ & $3 \mathrm{P} J=1-0$ & 492.2 & 0.3 \\
$\mathrm{CO}$ & $J=2-1$ & 230.5 & 7.9 \\
$\mathrm{CO}$ & $J=3-2$ & 345.8 & 22 \\
$\mathrm{CO}$ & $J=4-3$ & 461.0 & 41 \\
$\mathrm{CO}$ & $v=1, J=2-1$ & 228.4 & 2.5 \\
$\mathrm{CO}$ & $v=1, J=3-2$ & 342.6 & 11 \\
$\mathrm{CO}$ & $v=1, J=4-3$ & 456.8 & 27 \\
$\mathrm{SiO}$ & $v=0, J=5-4$ & 217.1 & 5.5 \\
$\mathrm{SiO}$ & $v=1, J=5-4$ & 215.6 & 3.5 \\
\hline
\end{tabular}

Notes. ${ }^{(a)}$ Peak flux densities have been calculated in the same way as for the Rydberg transition lines, with the assumption that the same line width $\left(8.5 \mathrm{~km} \mathrm{~s}^{-1}\right)$ and angular diameter (11 mas) apply to all.

\subsection{Predictions for radio lines}

As can be seen in Table 2 the ionisation degree is low $\left(\approx 10^{-4}\right)$ in the RTL region and the warm gas consists of predominantly neutral hydrogen, while the molecular fraction is dominated by $\mathrm{CO}$ at an abundance of $\approx 3 \times 10^{-4}$ with respect to hydrogen. The question is whether other atomic or molecular lines can be detected from the RTL region. The results presented in Table B.1 show that in the case of molecules, only $\mathrm{CO}$ and possibly $\mathrm{SiO}$ are viable candidates. Molecular nitrogen, $\mathrm{N}_{2}$, has a high abundance, but its lines are due to weak electric quadrupole transitions. Predictions are made for the LTE fluxes of some interesting atomic and molecular lines in Table 4.

In order to compare with our ALMA data, we re-analysed the molecular line data for the CCS component (see Sect. 2) using only the data obtained with the most extended configuration in Band 6, which therefore has the highest angular resolution, $\approx 0$ '.03, which is three times the size of the RTL region. The $\mathrm{S} / \mathrm{Ns}$ are high enough to determine the source sizes from the global line profiles, but not high enough to determine their brightness distributions as a function of velocity. We present the results for the $\mathrm{CO}(2-1)$ and $\mathrm{SiO}(5-4)$ lines because these species have high abundances in the RTL region, and for the $\mathrm{H}_{2} \mathrm{~S}\left(2_{20}-2_{11}\right)$ and $\mathrm{SO}_{2}\left(16_{3,13}-16_{2,14}\right)$ lines because they provide additional kinematic information. The $\mathrm{SO}_{2}$ line comes from a transition with an upper state energy corresponding to $148 \mathrm{~K}$, that is, a transition at high energy compared to those of the other molecular lines. We also added the upper limit obtained for the $\mathrm{CO}(v=1, J=3-2)$ line at an angular resolution of $\approx 0^{\prime \prime} .2$. The results are summarised in Table 5 .

All brightness distributions are larger than the RTL region by about a factor of ten or more (all sizes given here are FWHM of a Gaussian fitted to the brightness distribution and deconvolved with the beam). Except for the SiO line, the lines are reasonably fitted by a Gaussian line profile. For CO, the line width is substantially larger than that of the RTLs, while those of $\mathrm{H}_{2} \mathrm{~S}$ and $\mathrm{SO}_{2}$ are substantially smaller. Only the $\mathrm{SiO}$ line has a line width comparable to the RTL line width, but this line is clearly nonGaussian; it is double-peaked, a result corroborated by the profile of the ${ }^{29} \mathrm{SiO}(5-4)$ line. Therefore, the source sizes and the line profiles clearly show that the RTL region contributes only marginally, if at all, to the observed molecular lines. Only, the vibrationally excited $\mathrm{CO}$ rotational line deviates from this conclusion, but whether or not LTE is a good approximation for the vibrational excitation of $\mathrm{CO}$ is uncertain.

We also estimated the molecular flux densities within an aperture of $0^{\prime \prime} .03$ at the centre position. For $\mathrm{CO}$ and $\mathrm{SiO}$, these come out close to those estimated for the reference model (Table 4). This suggests that the RTL region may contribute significantly to these observed intensities. In contrast, the $\mathrm{H}_{2} \mathrm{~S}$ and $\mathrm{SO}_{2}$ lines must come from a region well outside the RTL region because the predicted abundances of these molecules are very low in the RTL region (Table B.1) and the lines are very narrow.

With the present data it is not easy to paint a consistent picture of the geometry and kinematics of the different line brightness distributions of the CCS component, including the RTL region. It would seem reasonable that the density and temperature of the gas declines with distance from the centre. This would explain why the more easily excited $\mathrm{CO}$ line emission is more extended than those of the other species. Chemistry may also play a role, restricting the three-atom molecules to the higher densities. The nature of the velocity field remains an open question because the observed lines (from $\mathrm{mm}$ wave to optical) show a number of different line profiles and line widths.

\section{Discussion}

\subsection{The origin of the RTL-region}

HD 101584 is believed to be in the aftermath of a CE process that ended about 800 years ago without a stellar merger (Olofsson et al. 2019). This resulted in the termination of the RGB evolution of the primary and only its core remains, while the low-mass companion lies at a distance of about 0.5 au from HD 101584. The complexity of this process, for which little observational information exists, and the absence of models for stars in this stage make it difficult to speculate about the origin of the warm gas. On the other hand, it does not appear unlikely that about $10^{-3} M_{\odot}$ of warm gas can leave the star at the end of the CE evolution. The gas is most likely stationary - although small effects of expansion and/or rotation cannot be excluded- but must be characterised by substantial micro-turbulence (especially if the optical lines discussed above also originate from this region). The exact process responsible for keeping the gas heated and turbulent remains unknown.

\subsection{A new tool for studying cooler stars?}

The detection of RTLs from low-ionisation-potential elements towards HD 101584, combined with the high sensitivity of ALMA, opens up the possibility of also detecting such lines towards other objects of cool to moderate temperature. In particular, red giants on the RGB or AGB may be detectable if they have enough gas in their immediate surroundings with temperatures around $3000 \mathrm{~K}$ and particle densities of the order $10^{12} \mathrm{~cm}^{-3}$. The studies of radio continuum emission from low-ionisation gas in the extended atmospheres of AGB stars indicate that this may very well be the case (Reid \& Menten 2007), Matthews et al. 2018, Vlemmings et al. 2019). To this category we can also add red supergiants. At temperatures in excess of about $4000 \mathrm{~K}$ we can expect the hydrogen lines to also become detectable. This is, for instance, the case for pre-PNe (without ionising radiation) and objects like yellow supergiants (e.g. Betelgeuse, O'Gorman et al. 2017) and hypergiants. The $\mathrm{H} 30 \alpha$ line has been reported in ALMA observations of the Fried Egg Nebula (Wallström 
Table 5. Molecular line characteristics of the CCS component.

\begin{tabular}{|lcccccc|}
\hline \hline Line & $\begin{array}{c}\theta_{\mathrm{b}}{ }^{a} \\
{\left[{ }^{\prime \prime} \times{ }^{\prime \prime}(\mathrm{PA})\right]}\end{array}$ & $\begin{array}{c}\theta_{\ell}{ }^{b} \\
{\left[{ }^{\prime \prime} \times \times^{\prime \prime}(\mathrm{PA})\right]}\end{array}$ & $\begin{array}{c}\text { Aperture } \\
{\left[{ }^{\prime \prime}\right]}\end{array}$ & $\begin{array}{c}S \\
{[\mathrm{mJy}]}\end{array}$ & $\begin{array}{c}\Delta v^{c} \\
{\left[\mathrm{~km} \mathrm{~s}^{-1}\right]}\end{array}$ & $\begin{array}{c}v_{\mathrm{c}} \\
{\left[\mathrm{km} \mathrm{s}^{-1}\right]}\end{array}$ \\
\hline $\mathrm{CO}(2-1)$ & $0.028 \times 0.026\left(40^{\circ}\right)$ & $0.180 \times 0.160\left(172^{\circ}\right)$ & 0.2 & 99 & 18.7 & 41.1 \\
& & - & 0.03 & 5.4 & 16.1 & 43.3 \\
$\mathrm{CO}(v=1,3-2)$ & $0.22 \times 0.18\left(-42^{\circ}\right)$ & - & 0.3 & $<5$ & - & - \\
$\mathrm{SiO}(5-4)^{d}$ & $0.030 \times 0.028\left(36^{\circ}\right)$ & $0.086 \times 0.075\left(136^{\circ}\right)$ & 0.1 & 24 & 12.3 & 43.9 \\
& & - & 0.03 & 4.3 & 10.7 & 44.0 \\
$\mathrm{H}_{2} \mathrm{~S}\left(2_{20}-2_{11}\right)$ & $0.030 \times 0.028\left(36^{\circ}\right)$ & $0.088 \times 0.081\left(44^{\circ}\right)$ & 0.1 & 31 & 3.4 & 42.0 \\
& & - & 0.03 & 4.6 & 3.2 & 42.3 \\
$\mathrm{SO}_{2}\left(16_{3,13}-16_{2,14}\right)$ & $0.030 \times 0.028\left(36^{\circ}\right)$ & - & 0.1 & 32 & 1.9 & 41.6 \\
\hline \hline
\end{tabular}

Notes. ${ }^{(a)}$ The synthesised beam size and its PA. ${ }^{(b)}$ FWHM of a Gaussian fit to the brightness distribution of the integrated line profile (and deconvolved with the beam). ${ }^{(c)}$ FWHM of a Gaussian fit to the line profile (deconvolved with the spectral resolution of $1.5 \mathrm{~km} \mathrm{~s}^{-1}$ ). ${ }^{(d)}$ This line is double-peaked and the results of the Gaussian fit are only indicative.

et al. 2017), which is associated with the yellow hypergiant star Henize 3-1379 (IRAS 17163-3907).

Close binaries - like HD 101584 - of various types are also likely to be of interest in this context. In this category are the symbiotic stars. Several such stars, notably RR Tel, show detectable free-free continuum emission at $\mathrm{cm}$ wavelengths. An interesting example in the high-mass regime is VV Cep, which is an eclipsing binary star system consisting of a red supergiant that fills its Roche lobe when closest to the companion, a Be star that is most likely on the main sequence (Hagen Bauer et al. 2008). Here substantial amounts of warm gas can be produced through shocks and the hot radiation field.

The lines due to Rydberg transitions may therefore provide independent information on the warm gas in the vicinity of moderate-temperature stellar objects, and hence provide constraints on the origin and heating of such regions. Rydberg transitions of heavy elements are also susceptible to strong Zeeman splitting, and consequently can be used to estimate stellar magnetic fields, a property that is notoriously difficult to measure for evolved stars (e.g. Vlemmings 2014).

\section{Conclusions}

We identified two lines towards the post-giant star HD 101584 that can be attributed to the Rydberg transitions $30 \alpha$ and $26 \alpha$ of neutral atoms of elements heavier than carbon. We used a model in strict LTE and a source geometry in the form of a geometrically thin disc to interpret the data and to identify the line carrier(s). A solution that fits all the observational constraints (i.e. the measured line flux densities, upper limits to the continuum flux densities, and the estimated size of the emitting region) includes a gas mass of about $10^{-3} M_{\odot}$, a density of about $10^{12} \mathrm{~cm}^{-3}$, a temperature of about $2800 \mathrm{~K}$, a size of $\approx 10 \mathrm{au}$, and a turbulent velocity field in the range $5.5-7.5 \mathrm{~km} \mathrm{~s}^{-1}$, assuming a distance of $1 \mathrm{kpc}$ and solar abundances of the elements. The element $\mathrm{Mg}$ contributes about $75 \%$ of the line fluxes. The contributions from $\mathrm{Na}, \mathrm{Al}$, and $\mathrm{Ca}$ lie in the range of 5-10\% each. The corresponding $\mathrm{H}$ and $\mathrm{C}$ lines are much weaker. Using the Zeeman effect, we set an upper limit to the average magnetic field in the line-emitting region of $1 \mathrm{G}$.

Further observations are required to determine in more detail the geometry and kinematics of the region where the Rydberg transition lines are formed. The gas appears to be stationary, and the process responsible for keeping the gas heated and turbulent over a region of about 10 au remains to be identified; it may be connected to processes active during the $\mathrm{CE}$ phase.
Although HD 101584 is not a standard object, we speculate that the types of lines discussed here should be detectable towards other moderately warm and evolved objects, in particular taking the sensitivity of ALMA into account, and serve as a new tool for their study.

Acknowledgements. We are grateful to the referee (Albert Zijlstra) for providing insightful and constructive comments on the paper. HO and WV acknowledge support from the Swedish Research Council. This paper makes use of the following ALMA data: ADS/JAO.ALMA\#2012.1.00248.S, \#2015.1.00078.S and \#2018.1.00753.S. ALMA is a partnership of ESO (representing its member states), NSF (USA) and NINS (Japan), together with NRC (Canada) and NSC and ASIAA (Taiwan) and KASI (Republic of Korea), in cooperation with the Republic of Chile. The Joint ALMA Observatory is operated by ESO, AUI/NRAO and NAOJ. HO acknowledges support from the Nordic ALMA Regional Centre (ARC) node based at Onsala Space Observatory. The Nordic ARC node is funded through Swedish Research Council grant No 2017-00648. This research has made use of the services of the ESO Science Archive Facility. Based on observations collected at the European Southern Observatory under ESO programme 266.D-5655(A).

\section{References}

Alexander, J. \& Gulyaev, S. 2016, ApJ, 828, 40

Asplund, M., Grevesse, N., Sauval, A. J., \& Scott, P. 2009, ARA\&A, 47, 481

Bachiller, R., Huggins, P. J., Martin-Pintado, J., \& Cox, P. 1992, A\&A, 256, 231

Bakker, E. J., Lamers, H. J. G. L. M., Waters, L. B. F. M., et al. 1996, A\&A, 307, 869

Bell, K. L. \& Berrington, K. A. 1987, Journal of Physics B Atomic Molecular Physics, 20, 801

Bohr, N. 1920, Zeitschrift fur Physik, 2, 423

Brault, J. \& Noyes, R. 1983, ApJ, 269, L61

Brocklehurst, M. \& Leeman, S. 1971, Astrophys. Lett., 9, 35

Brocklehurst, M. \& Seaton, M. J. 1972, MNRAS, 157, 179

Brown, R. L., Lockman, F. J., \& Knapp, G. R. 1978, ARA\&A, 16, 445

Carlsson, M. \& Rutten, R. J. 1992, A\&A, 259, L53

Chaisson, E. J. 1975, ApJ, 197, L65

Chaisson, E. J., Black, J. H., Dupree, A. K., \& Cesarsky, D. A. 1972, ApJ, 173, L131

Chang, E. 1987, Phys. Scr, 35, 792

Chang, E. S. \& Noyes, R. W. 1983, ApJ, 275, L11

Clark, T. A., Naylor, D. A., \& Davis, G. R. 2000a, A\&A, 357, 757

Clark, T. A., Naylor, D. A., \& Davis, G. R. 2000b, A\&A, 361, L60

Cox, P., Martin-Pintado, J., Bachiller, R., et al. 1995, A\&A, 295, L39

Draine, B. T. 2011, Physics of the Interstellar and Intergalactic Medium (Princeton University Press)

Dravskikh, Z. V. \& Dravskikh, A. 1964, Astr. Tsirk., 282, 2

Gaia Collaboration, Brown, A. G. A., Vallenari, A., et al. 2018, A\&A, 616, A1

Gaia Collaboration, Brown, A. G. A., Vallenari, A., et al. 2020, arXiv e-prints, arXiv:2012.01533

Gaia Collaboration, Prusti, T., de Bruijne, J. H. J., et al. 2016, A\&A, 595, A1

Gordon, M. A. \& Sorochenko, R. L., eds. 2002, Astrophysics and Space Science Library, Vol. 282, Radio Recombination Lines. Their Physics and Astronomical Applications (Springer Verlag)

Griem, H. 1967, ApJ, 148, 547 
Hagen Bauer, W., Gull, T. R., \& Bennett, P. D. 2008, AJ, 136, 1312

Hoang-Binh, D. \& van Regemorter, H. 1995, Journal of Physics B, Atomic Molecular Physics, 28, 3147

Höglund, B. \& Mezger, P. G. 1965, Science, 150, 339

Irwin, A. W. 1988, A\&AS, 74, 145

James, B. \& Aloisi, A. 2018, ApJ, 853, 124

John, T. L. 1975a, MNRAS, 172, 305

John, T. L. 1975b, MNRAS, 170, 5

John, T. L. 1994a, MNRAS, 266, 186

John, T. L. 1994b, MNRAS, 269, 871

John, T. L. 1994c, MNRAS, 269, 865

John, T. L. 1994d, A\&A, 282, 890

Kardashev, N. S. 1959, Soviet Ast., 3, 813

Kaulakys, B. 1984, Journal of Physics B, Atomic Molecular Physics, 17, 4485

Kipper, T. 2005, Baltic Astronomy, 14, 223

Kluska, J., Olofsson, H., Van Winckel, H., et al. 2020, A\&A, 642, A152

Lebouteiller, V., Kuassivi, \& Ferlet, R. 2005, A\&A, 443, 509

Lepp, S., Dalgarno, A., van Dishoeck, E. F., \& Black, J. H. 1988, ApJ, 329, 418

Lilley, A. E., Palmer, P., Penfield, H., \& Zuckerman, B. 1966, Nature, 211, 174

Martin-Pintado, J., Bachiller, R., Thum, C., \& Walmsley, M. 1989, A\&A, 215, L13

Matthews, L. D., Reid, M. J., Menten, K. M., \& Akiyama, K. 2018, AJ, 156, 15

McLaughlin, B. M., Stancil, P. C., Sadeghpour, H. R., \& Forrey, R. C. 2017a Journal of Physics B, Atomic Molecular Physics, 50, 209501

McLaughlin, B. M., Stancil, P. C., Sadeghpour, H. R., \& Forrey, R. C. 2017b, Journal of Physics B, Atomic Molecular Physics, 50, 114001

McMullin, J. P., Waters, B., Schiebel, D., Young, W., \& Golap, K. 2007, in Astronomical Society of the Pacific Conference Series, Vol. 376, Astronomical Data Analysis Software and Systems XVI, ed. R. A. Shaw, F. Hill, \& D. J. Bell, 127

Menzel, D. H. 1937, ApJ, 85, 330

Mitroy, J., Safronova, M. S., \& Clark, C. 2010, Journal of Physics B Atomic Molecular Physics, 43, 202001

Moomey, D., Federman, S. R., \& Sheffer, Y. 2012, ApJ, 744, 174

Murcray, F. J., Goldman, A., Murcray, F. H., et al. 1981, ApJ, 247, L97

O’Gorman, E., Kervella, P., Harper, G. M., et al. 2017, A\&A, 602, L10

Olofsson, H., Khouri, T., Maercker, M., et al. 2019, A\&A, 623, A153

Olofsson, H., Vlemmings, W. H. T., Bergman, P., et al. 2017, A\&A, 603, L2

Omont, A. 1977, Journal de Physique, 38, 1343

Pankonin, V., Thomasson, P., \& Barsuhn, J. 1977, A\&A, 54, 335

Parthasarathy, M. \& Pottasch, S. R. 1986, A\&A, 154, L16

Reid, M. J. \& Menten, K. M. 2007, ApJ, 671, 2068

Roelfsema, P. R., Goss, W. M., Pottasch, S. R., \& Zijlstra, A. 1991, A\&A, 251, 611

Sánchez Contreras, C., Báez-Rubio, A., Alcolea, J., Bujarrabal, V., \& MartínPintado, J. 2017, A\&A, 603, A67

Schöier, F. L., van der Tak, F. F. S., van Dishoeck, E. F., \& Black, J. H. 2005, A\&A, 432, 369

Stallcop, J. R. 1974, ApJ, 187, 179

Storey, P. J. \& Hummer, D. G. 1991, Computer Physics Communications, 66, 129

Tennyson, J., Yurchenko, S. N., Al-Refaie, A. F., et al. 2016, Journal of Molecular Spectroscopy, 327, 73

Thum, C., Neri, R., Báez-Rubio, A., \& Krips, M. 2013, A\&A, 556, A129

Towle, J. P., Feldman, P. A., \& Watson, J. G. 1996, ApJS, 107, 747

van de Hulst, H. C. 1945, Nederlandsch Tijdschrift voor Natuurkunde, 11, 210

van der Tak, F. F. S., Lique, F., Faure, A., Black, J. H., \& van Dishoeck, E. F. 2020, Atoms, 8, 15

van Hoof, P. A. M. 2000, MNRAS, 314, 99

van Hoof, P. A. M., Williams, R. J. R., Volk, K., et al. 2014, MNRAS, 444, 420

Vlemmings, W. H. T. 2014, in IAU Symposium, Vol. 302, Magnetic Fields throughout Stellar Evolution, ed. P. Petit, M. Jardine, \& H. C. Spruit, 389397

Vlemmings, W. H. T., Khouri, T., \& Olofsson, H. 2019, A\&A, 626, A81

Wadiak, E. J., Sarazin, C. L., \& Brown, R. L. 1983, ApJS, 53, 351

Wallström, S. H. J., Lagadec, E., Muller, S., et al. 2017, A\&A, 597, A99

Watson, J. K. G. 2006, Journal of Physics B Atomic Molecular Physics, 39, 1889 


\section{Appendix A: Elemental abundances}

The abundances are expressed on the standard logarithmic scale $[\mathrm{H}]=12.0$, so that the relative number densities are $\log \left(n_{\mathrm{X}} / n_{\mathrm{H}}\right)=Y(\mathrm{X})-Y(\mathrm{H})+\log d(\mathrm{X})$; see Table A.1. For Set 1 we adopt solar abundances and all $d(\mathrm{X}) \equiv 1.0)$. The depletion factors for Set 2 are typical of well-mixed, diffuse interstellar gas where refractory elements have already been condensed into dust. Only elements up to $\mathrm{Ni}$ with ionisation potentials lower than that of hydrogen are considered.

Table A.1. Adopted elemental abundances

\begin{tabular}{lrrl}
\hline \hline $\mathrm{X}$ & \multicolumn{1}{c}{$I / k_{\mathrm{B}}{ }^{a}$} & $Y(\mathrm{X})$ & $d(\mathrm{X})^{b}$ \\
& \multicolumn{1}{l}{]} & & \\
\hline $\mathrm{H}$ & 157803 & 12.00 & 1.0 \\
$\mathrm{C}$ & 130670 & 8.43 & 0.458 \\
$\mathrm{Na}$ & 59636 & 6.24 & 0.36 \\
$\mathrm{Mg}$ & 88731 & 7.60 & 0.112 \\
$\mathrm{Al}$ & 69462 & 6.45 & 0.00179 \\
$\mathrm{Si}$ & 94596 & 7.51 & 0.0479 \\
$\mathrm{P}$ & 121693 & 5.41 & 1.0 \\
$\mathrm{~S}$ & 120223 & 7.12 & 1.0 \\
$\mathrm{Cl}$ & 150483 & 5.50 & 0.324 \\
$\mathrm{~K}$ & 50371 & 5.03 & 0.078 \\
$\mathrm{Ca}$ & 70940 & 6.34 & 0.0001739 \\
$\mathrm{Ti}$ & 79237 & 4.95 & 0.0022 \\
$\mathrm{Fe}$ & 91704 & 7.50 & 0.00375 \\
$\mathrm{Ni}$ & 88657 & 6.22 & 0.00176 \\
\hline
\end{tabular}

Notes. ${ }^{(a)}$ The ionisation energy ( $k_{\mathrm{B}}$ is the Boltzmann constant). ${ }^{(b)}$ The depletion factor. Solar photospheric abundances are taken from Asplund et al. (2009). Depleted abundances follow the patterns in diffuse molecular clouds and are taken from Draine (2011), except for $\mathrm{Na}$ and $\mathrm{K}$ (Lepp et al. 1988), P (Lebouteiller et al. 2005, James \& Aloisi 2018), and $\mathrm{Cl}$ (Moomey et al. 2012).

\section{Appendix B: Chemistry}

Table B.1 lists the molecules that have been included in the chemistry and ionisation balance in the LTE models. For each molecule $\mathrm{X}$, the fractional abundance is given with respect to $\mathrm{H}$ in the reference model. The adopted dissociation energy $D_{0}$ is expressed in wavenumber units of $\mathrm{cm}^{-1}$ (one electron volt is $\left.\mathrm{eV}=8065.5439 \mathrm{~cm}^{-1}\right)$, and the source of the partition function is listed. For most of the included molecules, tables of partition functions were taken from the ExoMol Database (Tennyson et al. 2016.6 and used via interpolation. In a few cases, notably $\mathrm{H}_{2}, \mathrm{CO}$, and $\mathrm{N}_{2}$, the partition functions were calculated directly by summing over states listed in extensive spectroscopic tables compiled for the Leiden Atomic and Molecular Database for Astronomy (LAMDA) (Schöier et al. 2005, van der Tak et al. 2020|7.

Although 30 diatomic and triatomic molecules have been included in the equilibrium computation of ionisation, only a few of them have significant abundances. Notice that the primary solutions reflect conditions in which a small fraction of the hydrogen is in molecular form, while a significant fraction of the total carbon and oxygen is consumed in $\mathrm{CO}$. The relatively high abundances of $\mathrm{CO}, \mathrm{N}_{2}$, and $\mathrm{SiO}$ reflect their high dissociation energies. At $T \approx 2800 \mathrm{~K}$ and $n_{\mathrm{H}} \approx 10^{12} \mathrm{~cm}^{-3}$ in a gas of solar abundances, the presence of these most abundant molecules does

\footnotetext{
6 http://www. exomol.com/

7 https://home.strw.leidenuniv.nl/ moldata/
}

Table B.1. Molecules and their equilibrium abundances in the reference model

\begin{tabular}{lrrl}
\hline \hline $\mathrm{X}$ & $\mathrm{X} / \mathrm{H}$ & $\begin{array}{c}D_{0} \\
{\left[\mathrm{~cm}^{-1}\right]}\end{array}$ & Partition function \\
\hline $\mathrm{H}_{2}$ & -4.34 & 36118 & state sum \\
$\mathrm{H}_{2}^{+}$ & -17.88 & 21379 & state sum \\
$\mathrm{H}_{3}^{+}$ & -19.85 & 35270 & state sum \\
$\mathrm{CO}$ & -3.57 & 89463 & state sum \\
$\mathrm{H}^{-}$ & -12.35 & 6084 & one bound state \\
$\mathrm{MgH}$ & -12.74 & 10366 & ExoMol \\
$\mathrm{MgO}$ & -14.82 & 21777 & ExoMol \\
$\mathrm{SiO}$ & -6.46 & 66621 & ExoMol \\
$\mathrm{SiH}$ & -10.29 & 24358 & ExoMol \\
$\mathrm{N}_{2}$ & -4.64 & 78715 & state sum \\
$\mathrm{CN}$ & -9.38 & 62589 & ExoMol \\
$\mathrm{NO}$ & -8.66 & 52483 & ExoMol \\
$\mathrm{SO}$ & -11.84 & 43175 & state sum \\
$\mathrm{SO}$ & -19.29 & 45652 & ExoMol \\
$\mathrm{SH}$ & -9.77 & 29197 & ExoMol \\
$\mathrm{TiO}$ & -12.46 & 55488 & ExoMol \\
$\mathrm{TiO}_{2}$ & -18.60 & 50410 & Irwin $(1988)$ \\
$\mathrm{TiO}^{+}$ & -11.32 & 55492 & Black (in prep.) \\
$\mathrm{OH}$ & -7.48 & 35480 & ExoMol \\
$\mathrm{H}_{2} \mathrm{O}$ & -11.58 & 41241 & ExoMol \\
$\mathrm{HCN}$ & -12.33 & 43247 & ExoMol \\
$\mathrm{C}_{2}$ & -14.32 & 50370 & ExoMol \\
$\mathrm{CH}_{\mathrm{HCO}}$ & -11.84 & 28132 & ExoMol \\
$\mathrm{TiH}^{+}$ & -17.54 & 49075 & state sum \\
$\mathrm{H}_{2} \mathrm{~S}$ & -15.80 & 16776 & ExoMol \\
$\mathrm{FeH}$ & -12.64 & 30938 & ExoMol \\
$\mathrm{FeO}$ & -13.15 & 33715 & ExoMol \\
$\mathrm{CS}$ & -11.12 & 59322 & ExoMol \\
$\mathrm{CO}$ & -11.57 & 44399 & ExoMol \\
\hline & & &
\end{tabular}

have a small effect on the overall ionisation balance and thus the intensity of the free-free continuum emission. In addition, these models can be used to predict the intensity of molecular line emission that should accompany the atomic lines under the adopted assumptions about strict LTE and solar abundances.

\section{Appendix C: Additional line broadening}

Because Rydberg atoms interact strongly with electrons, other atoms, radiation, and magnetic fields, it is worthwhile exploring whether or not the line profiles contain additional information about the conditions in the emitting regions. This can be done through computation of theoretical line profiles based on the conditions in the reference model described above. In order to do this properly, the fine structure of the Rydberg transitions will have to be added.

\section{Appendix C.1: Fine structure}

Rydberg states of principal quantum number $n$ possess slightly non-degenerate fine structure in their orbital substates (quantum number $\ell=0$ to $n-1)$. Hence, a Rydberg transition $n^{\prime} \rightarrow n^{\prime \prime}$ is composed of many fine-structure components $\left(n^{\prime}, \ell^{\prime}\right) \rightarrow\left(n^{\prime \prime}, \ell^{\prime \prime}\right)$ that obey the selection rules $\ell^{\prime}=\ell^{\prime \prime} \pm 1$. Although the finestructure splitting is negligible at $n=25$ to 30 in hydrogen (Towle et al. 1996), atoms like $\mathrm{Mg}, \mathrm{Al}$, and $\mathrm{Ca}$ have parental ionic cores with rather high values of the static dipole polaris- 
ability, which gives rise to larger fine-structure splittings than in hydrogen. Thus, the $26 \alpha$ transition of each atomic emitter has 51 fine-structure components and the model profile for this transition is a blend of 408 lines for the atomic species considered in the reference model; see Table C.1 The same figures for the $30 \alpha$ transition are 59 and 472 . In the construction of a theoretical profile, each of the fine-structure components is weighted by the relative abundance of the atomic species and by an intensity factor, which is proportional to the upper-state statistical weight multiplied by the spontaneous transition probability.

We computed the energy of each state of each atom, labelled $(n, \ell)$, from the solution of the Dirac equation for a hydrogenic atom with $n \gg 1$ as presented in equations 2 through 4 of Towle et al. (1996). An additional term, $-\alpha_{\mathrm{d}} P(n, \ell)$, is included to account for the polarisation of the ionic core, where $P(n, \ell)$ is a function of $n$ and $\ell$, and $\alpha_{\mathrm{d}}$ is the electric dipole polarisability (Chang \& Noyes 1983; Chang 1987). In H itself, with a bare proton core, the polarisability vanishes and this term is zero. In $\mathrm{Mg}$, on the other hand, the $\mathrm{Mg}^{+}$core has $\alpha_{\mathrm{d}}=33.05 \pm 0.03 a_{0}^{3}$ (atomic units, where $a_{0}$ is the Bohr radius (see Mitroy et al. 2010)). We computed an $\ell$-resolved line list for each transition $n^{\prime} \rightarrow n^{\prime \prime}$. The number of fine-structure components (distinct lines) in an $\alpha$ transition is $2 n^{\prime \prime}-1$, where double-prime indicates the lower state. The spontaneous transition probabilities (Einstein $A$-coefficients) for these hydrogenic transitions were computed through use of the computer program of Storey \& Hummer (1991). We assume that pure L-S coupling applies. For Rydberg states in the limit of high- $n$, all atoms are assumed to behave like degenerate hydrogenic spin-doublets of total angular momentum $j=\ell \pm 1 / 2$. The degeneracy is lifted in the presence of external fields, as we explain below in the section on Zeeman splitting. In the absence of external fields, the blending of lines of different atoms and the overlap of their fine-structure components broaden the line profile and make it slightly asymmetrical. The fine-structure splittings in the $26 \alpha$ and $30 \alpha$ transitions correspond to only a few tenths of a $\mathrm{km} \mathrm{s}^{-1}$, and hence will not contribute substantially to the line widths measured here.

\section{Appendix C.2: Collisional broadening}

Atoms in Rydberg states interact with electrons and neutral $\mathrm{H}$ atoms through elastic and inelastic collisions that perturb their energies and thus broaden their line profiles. This collisional broadening depends on the densities of the collision partners and on the kinetic temperature. In ionised nebulae, recombination lines suffer collisional broadening mainly with electrons and ions. In contrast, our reference model is only weakly ionised, with $n_{\mathrm{e}} / n_{\mathrm{H}}=3.4 \times 10^{-5}$; as a result, neutral hydrogen and electrons are the most important collision partners.

The electron-impact broadening of nebular recombination lines has been discussed in the literature; unfortunately, most of that work is not applicable here. Watson (2006) presented a formula for the collisional broadening based on the classic work of Griem (1967). As pointed out by Alexander \& Gulyaev (2016), that formula contains errors. Beyond that, Griem's approximation was originally intended for $\mathrm{cm}$ - and dm-wave transitions with $n>100$; the approximation breaks down when $n<70$ and is thus not appropriate for the transitions of interest here. Brocklehurst \& Leeman (1971) computed the electronhydrogen cross-sections and rates numerically, and evaluated the line-broadening without the restrictive assumptions of semiclassical theory. Their results could be approximated by a simple fitting formula for the collisional line width of $\alpha$ transitions,

$$
\frac{\Delta v_{\mathrm{e}}}{v_{0}}=1.43 \times 10^{-5}\left(\frac{n}{100}\right)^{7.4}\left(\frac{10^{4} \mathrm{~K}}{T}\right)^{0.1}\left(\frac{n_{\mathrm{e}}}{10^{4} \mathrm{~cm}^{-3}}\right)
$$

where $T$ and $n_{\mathrm{e}}$ are the temperature and number density of electrons, respectively (Brocklehurst \& Seaton 1972). Because the original computations did not extend to $n<109$ and because the dependence on $n$ is so steep, it is not certain that this formula is accurate at $n=26$ and 30 . If we simply apply the formula for $T=2805 \mathrm{~K}$ and $n_{\mathrm{e}}=2.8 \times 10^{7} \mathrm{~cm}^{-3}$, as in the reference model, then the electron-impact widths (FWHM) are $\Delta v_{\mathrm{e}}=0.63$ and $1.83 \mathrm{~km} \mathrm{~s}^{-1}$ for $n=26$ and 30 , respectively. We note that the collisional broadening produces a Lorentzian line shape rather than a Gaussian. Moreover, if electron-impact broadening were to dominate, the width of the $30 \alpha$ line would be almost three times larger than that of $26 \alpha$ in velocity units according to the formula of Brocklehurst \& Seaton (1972).

Collisional broadening by neutral atoms has also been considered, particularly in connection with laboratory experiments on Rydberg atoms in gases of noble or alkali atoms (Omont 1977, Kaulakys 1984). Neutral-atom collisions are also important in line-broadening in stellar atmospheres. Specifically, Hoang-Binh \& van Regemorter (1995) applied the impulse approximation to compute the broadening of mid-infrared lines of $\mathrm{Mg}$ I by atomic hydrogen in the photosphere of the Sun, where these lines are observed in emission at wavelengths near $12 \mu \mathrm{m}$. These are also Rydberg transitions, but at the relatively low values of the principal quantum number involved, $n=5,6$, and 7 , the orbital and spin-orbit fine structures are well resolved. If we adapt the theoretical widths calculated by Omont (1977) to the case of $\mathrm{H}$ on $\mathrm{Mg}$, we estimate $\Delta v_{\mathrm{H}} \approx 1.8 \mathrm{MHz}$ at both $n=26$ and 30 , corresponding to velocity widths $\Delta v_{\mathrm{H}}=1.5$ and $2.2 \mathrm{~km} \mathrm{~s}^{-1}$, respectively, in the reference model. Even though the neutralneutral interactions are much weaker than electron-neutral interactions involving Rydberg states, the estimated contributions to the line broadening are comparable in our case because of the low electron fraction.

In both the cases of electron and neutral collisions, the collisional broadening should be re-computed numerically with modern methods, because both elastic and inelastic collisions are important and earlier approximations at $n>100$ are known to break down when $n \sim 20$ to 30, but this is beyond the scope of this paper. The above estimates suggest that the collisional broadening might become noticeable if the line profile can be observed with sufficiently high $\mathrm{S} / \mathrm{N}$ to reveal Lorentzian wings. However, the existing data show line profiles that are fit very well by singleGaussian functions, meaning that, even with the uncertainties in the theoretical line widths, the current data on HD 101584 suggest that the neutral density in the emitting region cannot be as large as $10^{13} \mathrm{~cm}^{-3}$, otherwise the Lorentzian wings would be apparent. This kind of limit on density is not model dependent; moreover, it could be refined with improved theory.

\section{Appendix C.3: Radiation damping}

Atoms in Rydberg states also interact with electromagnetic radiation. The natural line broadening (radiation damping) via spontaneous transitions can be further enhanced through stimulated emission and absorption in the ambient radiation field. Considering a transition from upper state $\mathrm{u}$ to lower state 1 , the energies of these states are diffuse because both states have finite lifetimes. The Lorentzian FWHM of the radiation damping is 
$\Delta v_{\mathrm{rad}}=\gamma /(2 \pi)$ where

$$
\begin{aligned}
\gamma_{\mathrm{ul}}= & \sum_{\mathrm{i}<\mathrm{u}}\left(A_{\mathrm{ui}}+\bar{J}_{\mathrm{ui}} B_{\mathrm{ui}}\right)+\sum_{\mathrm{k}>\mathrm{u}} \bar{J}_{\mathrm{ku}} B_{\mathrm{ku}}+\sum_{\mathrm{i}^{\prime}<1}\left(A_{\mathrm{li}^{\prime}}+\bar{J}_{\mathrm{li}^{\prime}} B_{\mathrm{li}^{\prime}}\right)+ \\
& +\sum_{\mathrm{k}^{\prime}>1} \bar{J}_{\mathrm{k}^{\prime} 1} B_{\mathrm{k}^{\prime} 1},
\end{aligned}
$$

and $A_{\mathrm{ik}}$ is the spontaneous transition probability for transition $\mathrm{i} \rightarrow \mathrm{k}$, and $\bar{J}_{\mathrm{ik}}$ the angle-averaged intensity of radiation at the frequency $v_{\mathrm{ik}}$ of the transition. The Einstein coefficients for stimulated emission, $B_{\mathrm{ik}}=A_{\mathrm{ik}} /\left(2 \mathrm{~h} v^{3} / c^{2}\right)$, and absorption, $B_{\mathrm{ki}}=g_{\mathrm{i}} B_{\mathrm{ik}} / g_{\mathrm{k}}$ are related to $A_{\mathrm{ik}}$ and to the statistical weights of the upper and lower states $g_{\mathrm{i}}$ and $g_{\mathrm{k}}$. When the radiation can be represented by a blackbody diluted by a geometrical factor $w \leq 1$, then

$$
\bar{J}_{\mathrm{ik}}=w B_{v_{\mathrm{ik}}}\left(T_{\mathrm{rad}}\right)=\frac{w 2 h v_{\mathrm{ik}}^{3} / c^{2}}{\exp \left(h v_{\mathrm{ik}} / k_{\mathrm{B}} T_{\mathrm{rad}}\right)-1},
$$

in terms of the Planck function $B_{v}$ at temperature $T_{\text {rad }}$. Thus, the damping coefficient can be re-written

$$
\begin{aligned}
\gamma_{\mathrm{ul}}= & \sum_{\mathrm{i}<\mathrm{u}} A_{\mathrm{ui}}\left(1+b\left(v_{\mathrm{ui}}\right)\right)+\sum_{\mathrm{k}>\mathrm{u}} A_{\mathrm{ku}} b\left(v_{\mathrm{ku}}\right) \frac{g_{\mathrm{k}}}{g_{\mathrm{u}}} \\
& +\sum_{\mathrm{i}^{\prime}<1} A_{\mathrm{li}^{\prime}}\left(1+b\left(v_{\mathrm{li}^{\prime}}\right)\right)+\sum_{\mathrm{k}^{\prime}>1} A_{\mathrm{k}^{\prime} \mathrm{l}} b\left(v_{\mathrm{k}^{\prime} 1}\right) \frac{g_{\mathrm{k}}^{\prime}}{g_{1}},
\end{aligned}
$$

where $b(v)=w /\left(\exp \left(h v / k_{\mathrm{B}} T_{\mathrm{rad}}\right)-1\right)$. Our model of the lineemitting region assumes strict LTE at the kinetic temperature $T$. This means that $w=1$ and $T_{\text {rad }}=T$ should be a good approximation at frequencies less than that of the $26 \alpha$ transition, owing to the inverse frequency dependence of the predominant opacity source. However, some of the stimulated transitions occur at much higher frequencies where the emitting region may be transparent to radiation from the much hotter visible photosphere of HD 101584 , for which $w<1$ and $T_{\text {rad }} \sim 8500 \mathrm{~K}$.

The computation of $\Delta v_{\text {rad }}$ is straightforward for hydrogenic transition frequencies and transition probabilities and is done here by summing all rates for states up to $n=1000$. The radiation damping of the $26 \alpha$ and $30 \alpha$ lines at $T_{\text {rad }}=2805 \mathrm{~K}$ and $w=1$ gives $\Delta v_{\text {rad }}$ corresponding to 0.072 and $0.083 \mathrm{~km} \mathrm{~s}^{-1} \mathrm{~km} \mathrm{~s}^{-1}$, respectively. Even in the case of the hot photosphere $T_{\mathrm{rad}}=8500 \mathrm{~K}$ shining through the emission region at $w=1 / 2$, the damping widths would be increased by only about $50 \%$. In either case, the radiation damping is negligible in comparison with other broadening mechanisms. In general, this broadening effect on Rydberg transitions might be most important at very low radio frequencies $(n>200)$ in gas exposed to intense non-thermal continuum radiation, as in the narrow-line regions of quasars (cf. Wadiak et al. 1983).

Both the collisional line broadening and the radiation damping produce Lorentzian profiles. Two Lorentz functions combine to form a new Lorentz function whose width is simply the sum of the first two Lorentz widths. The total profile is obtained by a convolution of the final Lorentz function with the profile for the blend.

\section{Appendix C.4: Magnetic fields and Zeeman broadening}

Magnetic fields can cause a line broadening in radio and $\mathrm{mm}-$ wave RTLs through the Zeeman effect. The blending of lines of different emitting atoms, each with the orbital fine structure

\begin{tabular}{|c|c|c|c|c|c|}
\hline \multirow[t]{3}{*}{ Atom } & \multirow{3}{*}{$\begin{array}{l}\text { Intensity }^{a} \\
\text { weighting }\end{array}$} & \multicolumn{4}{|c|}{ Line components } \\
\hline & & \multicolumn{2}{|r|}{$26 \alpha$} & \multicolumn{2}{|c|}{$30 \alpha$} \\
\hline & & $n, \ell$ & $n, \ell, j, m_{j}$ & $n, \ell$ & $n, \ell, j, m_{j}$ \\
\hline${ }^{24} \mathrm{Mg}$ & 0.5825 & 51 & 3900 & 59 & 5220 \\
\hline${ }^{25} \mathrm{Mg}$ & 0.0737 & 51 & 3900 & 59 & 5220 \\
\hline${ }^{26} \mathrm{Mg}$ & 0.0812 & 51 & 3900 & 59 & 5220 \\
\hline $\mathrm{Na}$ & 0.0662 & 51 & 3900 & 59 & 5220 \\
\hline $\mathrm{Al}$ & 0.1060 & 51 & 3900 & 59 & 5220 \\
\hline $\mathrm{Ca}$ & 0.0832 & 51 & 3900 & 59 & 5220 \\
\hline $\mathrm{Si}$ & 0.0458 & 51 & 3900 & 59 & 5220 \\
\hline $\mathrm{Fe}$ & 0.0189 & 51 & 3900 & 59 & 5220 \\
\hline Total & & 408 & 31200 & 472 & 41760 \\
\hline
\end{tabular}
described above, is further complicated in the presence of external fields. A one-electron, hydrogenic state with spin $s=1 / 2$, labelled by the quantum numbers $n, \ell$, and $j$ in L-S coupling, has
Table C.1. Blending and fine structure

Notes. (a) The weighting factors reflect the equilibrium abundances and level populations of the neutral atoms in the reference model. Magnesium isotopes are assumed to be present in the same relative amounts as in standard terrestrial material, (0.79:0.10:0.11) for isotopic masses (24:25:26). Columns 3-6 list the numbers of fine-structure components, without and with an external magnetic field. These are summed in the last line of the table.

a pair of degenerate levels for each $j=\ell \pm 1 / 2$. The degeneracy, that is, the number of unresolved sublevels, is $2 j+1$. A magnetic field of strength $B$ lifts this degeneracy, and yields splittings in the weak-field limit of the Zeeman effect given by

$E\left(n, \ell, j, m_{j}\right)=E(n, l)+g \mu_{\mathrm{B}} B m_{j}$,

where $m_{j}=-j,-j+1, \ldots,+j-1,+j$. In L-S coupling the Landé $g$-factor is

$g=1+\frac{j(j+1)-\ell(\ell+1)+s(s+1)}{2 j(j+1)}$,

and $g \approx 1$ for the levels of high $j$ and $\ell$ that contribute most to the line profile. We note that $\mu_{\mathrm{B}} B / h=1.3996 B \mathrm{MHz}$ ( $B$ is in the unit of gauss), so that a field strength of the order of $B \approx 1 \mathrm{G}$ can produce a splitting of the order of $1 \mathrm{MHz}$, which may be significant in the lines of interest here.

\section{Appendix C.5: The blended line profile}

We have computed theoretical line profiles for the $26 \alpha$ and $30 \alpha$ transitions taking into account all of the effects of blending and broadening. Eight atomic species are included, as listed in Table C.1. The computed model profile represents the weighted sum of the scaled optical depths of the various $(n, \ell)$ line components. Each line component is broadened by a Gaussian of total Doppler width $\Delta v$. The thermal width is calculated separately for each atom, because it depends upon the atomic mass, while a single value of the micro-turbulent width was assumed for all.

Further, we computed a series of model profiles with several values of the damping width, $\Gamma$, the FWHM of the Lorentzian function that describes the combined effect of collisional broadening and radiation damping. The total profile is a convolution of the blend with a Lorentzian function of width $\Gamma$. The collisional broadening term scales directly with density. As discussed above, there is some uncertainty about the collisional broadening coefficients: if we adopt the nominal values, then the total damping is $\Gamma=2.63$ and $3.28 \mathrm{MHz}$ for $26 \alpha$ and $30 \alpha$, respectively, for the reference model. With this choice, the convolved profile has a Doppler core that can be fit rather well by a Gaussian of $\Delta v=8.5 \mathrm{~km} \mathrm{~s}^{-1}$, like the observed profile, provided that the turbulent width is $\Delta v_{\text {turb }}=5.5 \mathrm{~km} \mathrm{~s}^{-1}$ (in contrast to the best model 


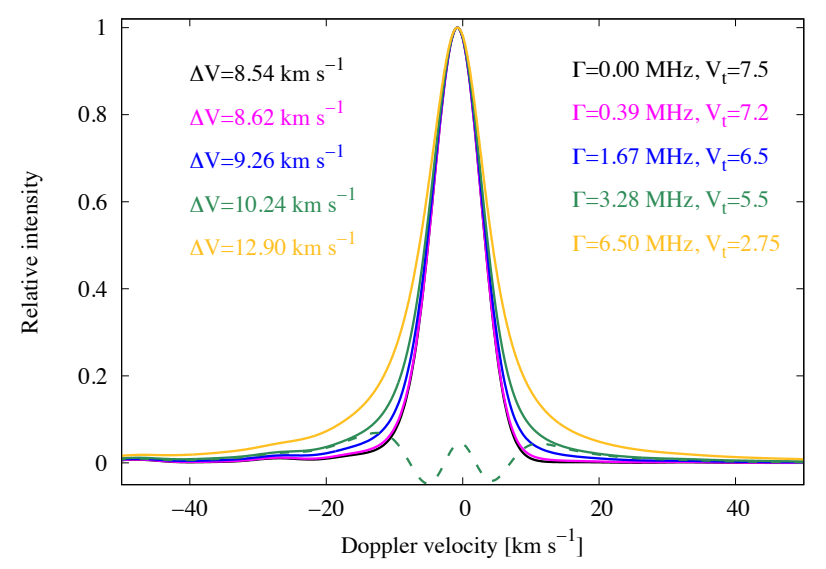

Fig. C.1. Series of model profiles of the $30 \alpha$ line for different values of the Lorentzian width $\Gamma$ and turbulent widths that give $\Delta v=8.5 \mathrm{~km} \mathrm{~s}^{-1}$ in the $26 \alpha$ line. The fitted Gaussian widths are indicated: they exceed the observed width when $\Gamma>1.7 \mathrm{MHz}$. The deviation from a single Gaussian is shown for the nominal case as a dashed green curve.

with no damping wings that requires $\Delta v_{\text {turb }}=7.5 \mathrm{~km} \mathrm{~s}^{-1}$ ). The deviation from a Gaussian profile is modest, and reaches about $7 \%$ of the peak intensity in the blue wing. It therefore appears that the collisional broadening effect will not be conspicuous in the analysis of only one line.

The situation becomes more interesting if the electronimpact collisional broadening does indeed have the strong $n$ dependence predicted by the existing theory at higher values of $n$. In that case, the best-fitting Gaussian width of the $30 \alpha$ transition is expected to be somewhat different from that of the $26 \alpha$ transition. Figure C. 1 shows the corresponding $30 \alpha$ profiles for the conditions that yield effective line widths of $8.5 \mathrm{~km} \mathrm{~s}^{-1}$ in the $26 \alpha$ line. Here, the nominal collisional broadening of the $30 \alpha$ line gives a profile with a best-fitting Gaussian width that is clearly larger, $\Delta v=10.2 \mathrm{~km} \mathrm{~s}^{-1}$. The collisional broadening width would need to be a factor of two times smaller in order for the effective widths of the two transitions to be approximately the same, if both lines are truly formed at the same density and temperature and turbulent width. This factor of two is within the uncertainty of the extrapolated theory of collisional broadening. On the other hand, collisional broadening two times larger than the nominal case would yield noticeable Lorentzian wings and a $50 \%$ difference in fitted FWHM between the two transitions. These model profiles were computed with reference to an LTE excitation model with density $n_{\mathrm{H}} \approx 8 \times 10^{11} \mathrm{~cm}^{-3}$. Thus it appears that the observed line profiles can already rule out densities higher than $n_{\mathrm{H}} \gtrsim 3 \times 10^{12} \mathrm{~cm}^{-3}$, independent of excitation models.

Finally, we have computed models with fully expressed Zeeman splitting for several combinations of magnetic field strength $B$ (assumed uniform through the source), turbulent width $\Delta v_{\text {turb }}$, and damping width $\Gamma$. A few examples of Zeeman-broadened profiles are shown in Fig. C.2. The combination of turbulence and damping tends to smooth out the structure of the Zeeman triplet when $B<2 \mathrm{G}$, so that the $26 \alpha$ profiles can all be made to approach Gaussian shapes with $\Delta v=8.5 \mathrm{~km} \mathrm{~s}^{-1}$ when the damping is small and $B \leq 1.5 \mathrm{G}$ ). However, the corresponding $30 \alpha$ profiles have Gaussian-fitted widths $\Delta v \geq 9.0 \mathrm{~km} \mathrm{~s}^{-1}$ when $B \geq 1.0 \mathrm{G}$. As the observed line widths of the two transitions are approximately equal, this suggests an upper limit to the field strength of $1 \mathrm{G}$ when the damping is small. The observed width of the $30 \alpha$ transition sets a strong upper limit of $B<2 \mathrm{G}$, because the fitted width of the computed profile is $9.2 \mathrm{~km} \mathrm{~s}^{-1}$ even

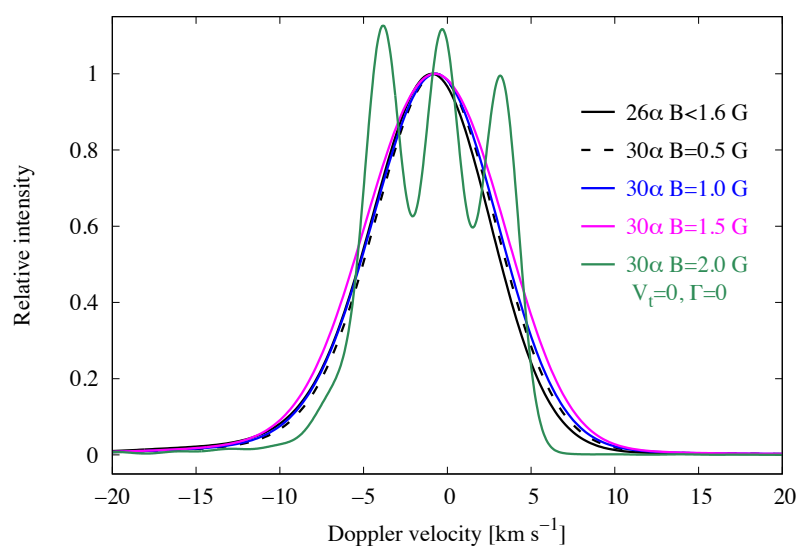

Fig. C.2. Series of model profiles including Zeeman splitting. Examples with small damping $(\Gamma=0.34 \mathrm{MHz}$ in $26 \alpha)$ are shown in black. The structure of the $26 \alpha$ profile is smoothed by turbulence and damping to approximate a Gaussian shape with $B$ in the range 0.5 to $1.6 \mathrm{G}$. The corresponding $30 \alpha$ profile is measurably broader, $>9.0 \mathrm{~km} \mathrm{~s}^{-1}$ when $B>1 \mathrm{G}$. A strong upper limit of $B<2 \mathrm{G}$ is implied by the fact that the $30 \alpha$ width is too large even when turbulence and damping are turned off, which then leaves the Zeeman triplet resolved (green curve).

when $\Delta v_{\text {turb }}=0$ and $\Gamma=0$ at $B=2 \mathrm{G}$. Again, the strong limit on magnetic field is independent of any excitation model, and is insensitive to uncertainties in the collisional broadening theory, but it depends on the field geometry.

\section{Appendix D: Dependences on geometry and distance}

Additional models were computed by us in order to explore the sensitivity of the solutions to the geometrical parameter $\rho$ and the distance $D$. We considered values of $\rho$ from 0.05 to 2.0 and distance ranges from 0.5 to $2.0 \mathrm{kpc}$. All models were constrained to fit the observed results at $232 \mathrm{GHz}: \theta=11.0$ mas, $S_{v}(\mathrm{X} 30 \alpha)=7.3 \mathrm{mJy}$, and $S_{\mathrm{c}}(232)=7.5 \mathrm{mJy}$. Details of additional models are summarised in Tables D.1 and D.3.

The models all behave similarly and the differences in bestfitting temperature (within $100 \mathrm{~K}$ ) and total density (within a factor of 3) are relatively small. The primary solutions all have continuum optical depths of the order of unity at $232 \mathrm{GHz}$, and the $\mathrm{X} 30 \alpha$ line optical depths for each contributing element are small enough that simply combining them in the optically thin limit is a good approximation.

\section{Appendix E: Optical lines}

Table E.1 shows a comparison with integrated line fluxes predicted by the reference model, including a correction for extinction. The simple equilibrium model was not tuned in any way to match the optical spectrum. The model nicely reproduces the integrated fluxes of the two $\mathrm{Ca}$ II forbidden lines. It under-predicts the 6300 and $6363 \AA$ lines of O I by a factor of six and the two C I lines by a much larger factor. However, the amounts of atomic $\mathrm{O}$ and $\mathrm{C}$ in the gas are very sensitive to the abundance of $\mathrm{CO}$, which in turn depends quadratically on density and exponentially on temperature in equilibrium. In the model, all of the tabulated lines have a peak optical depth $\tau<0.7$. Weak and forbidden lines were selected for a preliminary analysis because lines of higher optical depth would require a much more sophisticated treatment of radiative transfer through an extended atmosphere. 
Table D.1. Models reflecting a larger range of parameter space ${ }^{a}$.

\begin{tabular}{|c|c|c|}
\hline Property & & Primary solution \\
\hline$D$ & {$[\mathrm{kpc}]$} & 1.0 \\
\hline$\rho$ & & 0.1 \\
\hline$T$ & {$[\mathrm{~K}]$} & 2805 \\
\hline$n(\mathrm{H})$ & {$\left[\mathrm{cm}^{-3}\right]$} & $8.2 \times 10^{11}$ \\
\hline$R$ & [au] & 5.5 \\
\hline$\tau_{\ell}(232 \mathrm{GHz})$ & & 1.2 \\
\hline$\tau_{\mathrm{c}}(232 \mathrm{GHz})$ & & 1.3 \\
\hline$n(e)$ & {$\left[\mathrm{cm}^{-3}\right]$} & $2.8 \times 10^{7}$ \\
\hline$M_{\text {gas }}$ & {$\left[\mathrm{M}_{\odot}\right]$} & $7.2 \times 10^{-4}$ \\
\hline$n\left(\mathrm{H}_{2}\right)$ & {$\left[\mathrm{cm}^{-3}\right]$} & $3.7 \times 10^{7}$ \\
\hline$n(\mathrm{CO})$ & {$\left[\mathrm{cm}^{-3}\right]$} & $2.2 \times 10^{8}$ \\
\hline$n(\mathrm{OH})$ & {$\left[\mathrm{cm}^{-3}\right]$} & $2.7 \times 10^{4}$ \\
\hline$n(\mathrm{SiO})$ & {$\left[\mathrm{cm}^{-3}\right]$} & $2.8 \times 10^{5}$ \\
\hline$n\left(\mathrm{~N}_{2}\right)$ & {$\left[\mathrm{cm}^{-3}\right]$} & $1.9 \times 10^{7}$ \\
\hline$D$ & {$[\mathrm{kpc}]$} & 1.0 \\
\hline$\rho$ & & 0.05 \\
\hline$T$ & {$[\mathrm{~K}]$} & 2845 \\
\hline$n(\mathrm{H})$ & {$\left[\mathrm{cm}^{-3}\right]$} & $1.1 \times 10^{12}$ \\
\hline$R$ & [au] & 5.5 \\
\hline$\tau_{\ell}(232 \mathrm{GHz})$ & & 1.2 \\
\hline$\tau_{\mathrm{c}}(232 \mathrm{GHz})$ & & 1.3 \\
\hline$n(e)$ & {$\left[\mathrm{cm}^{-3}\right]$} & $3.9 \times 10^{7}$ \\
\hline$M_{\text {gas }}$ & {$\left[\mathrm{M}_{\odot}\right]$} & $4.6 \times 10^{-4}$ \\
\hline$n\left(\mathrm{H}_{2}\right)$ & {$\left[\mathrm{cm}^{-3}\right]$} & $5.2 \times 10^{7}$ \\
\hline$n(\mathrm{CO})$ & {$\left[\mathrm{cm}^{-3}\right]$} & $3.0 \times 10^{8}$ \\
\hline$n(\mathrm{OH})$ & {$\left[\mathrm{cm}^{-3}\right]$} & $4.1 \times 10^{4}$ \\
\hline$n(\mathrm{SiO})$ & {$\left[\mathrm{cm}^{-3}\right]$} & $3.4 \times 10^{5}$ \\
\hline$n\left(\mathrm{~N}_{2}\right)$ & {$\left[\mathrm{cm}^{-3}\right]$} & $2.5 \times 10^{7}$ \\
\hline$D$ & {$[\mathrm{kpc}]$} & 1.0 \\
\hline$\rho$ & & 0.2 \\
\hline$T$ & {$[\mathrm{~K}]$} & 2770 \\
\hline$n(\mathrm{H})$ & {$\left[\mathrm{cm}^{-3}\right]$} & $6.0 \times 10^{11}$ \\
\hline$R$ & [au] & 5.5 \\
\hline$\tau_{\ell}(232 \mathrm{GHz})$ & & 1.3 \\
\hline$\tau_{\mathrm{c}}(232 \mathrm{GHz})$ & & 1.3 \\
\hline$n(e)$ & {$\left[\mathrm{cm}^{-3}\right]$} & $2.0 \times 10^{7}$ \\
\hline$M_{\text {gas }}$ & {$\left[\mathrm{M}_{\odot}\right]$} & $1.2 \times 10^{-3}$ \\
\hline$n\left(\mathrm{H}_{2}\right)$ & {$\left[\mathrm{cm}^{-3}\right]$} & $2.5 \times 10^{7}$ \\
\hline$n(\mathrm{CO})$ & {$\left[\mathrm{cm}^{-3}\right]$} & $1.6 \times 10^{8}$ \\
\hline$n(\mathrm{OH})$ & {$\left[\mathrm{cm}^{-3}\right]$} & $1.8 \times 10^{4}$ \\
\hline$n(\mathrm{SiO})$ & {$\left[\mathrm{cm}^{-3}\right]$} & $2.3 \times 10^{5}$ \\
\hline$n\left(\mathrm{~N}_{2}\right)$ & {$\left[\mathrm{cm}^{-3}\right]$} & $1.4 \times 10^{7}$ \\
\hline$D$ & {$[\mathrm{kpc}]$} & 1.0 \\
\hline$\rho$ & & 1.0 \\
\hline$T$ & {$[\mathrm{~K}]$} & 2690 \\
\hline$n(\mathrm{H})$ & {$\left[\mathrm{cm}^{-3}\right]$} & $2.9 \times 10^{11}$ \\
\hline$R$ & [au] & 5.5 \\
\hline$\tau_{\ell}(232 \mathrm{GHz})$ & & 1.4 \\
\hline$\tau_{\mathrm{c}}(232 \mathrm{GHz})$ & & 1.4 \\
\hline$n(e)$ & {$\left[\mathrm{cm}^{-3}\right]$} & $8.6 \times 10^{6}$ \\
\hline$M_{\mathrm{gas}}$ & {$\left[\mathrm{M}_{\odot}\right]$} & $5.7 \times 10^{-4}$ \\
\hline$n\left(\mathrm{H}_{2}\right)$ & {$\left[\mathrm{cm}^{-3}\right]$} & $1.0 \times 10^{7}$ \\
\hline$n(\mathrm{CO})$ & {$\left[\mathrm{cm}^{-3}\right]$} & $7.8 \times 10^{7}$ \\
\hline$n(\mathrm{OH})$ & {$\left[\mathrm{cm}^{-3}\right]$} & $7.4 \times 10^{3}$ \\
\hline$n(\mathrm{SiO})$ & {$\left[\mathrm{cm}^{-3}\right]$} & $1.5 \times 10^{5}$ \\
\hline$n\left(\mathrm{~N}_{2}\right)$ & {$\left[\mathrm{cm}^{-3}\right]$} & $7.4 \times 10^{6}$ \\
\hline
\end{tabular}

Table D.2. Models reflecting a larger range of parameter space, continued.

\begin{tabular}{|c|c|c|}
\hline Property & & Primary solution \\
\hline$D$ & {$[\mathrm{kpc}]$} & 1.0 \\
\hline$\rho$ & & 2.0 \\
\hline$T$ & {$[\mathrm{~K}]$} & 2654 \\
\hline$n(\mathrm{H})$ & {$\left[\mathrm{cm}^{-3}\right]$} & $2.1 \times 10^{11}$ \\
\hline$R$ & [au] & 5.5 \\
\hline$\tau_{\ell}(232 \mathrm{GHz})$ & & 1.4 \\
\hline$\tau_{\mathrm{c}}(232 \mathrm{GHz})$ & & 1.4 \\
\hline$n(e)$ & {$\left[\mathrm{cm}^{-3}\right]$} & $6.0 \times 10^{6}$ \\
\hline$M_{\mathrm{gas}}$ & {$\left[\mathrm{M}_{\odot}\right]$} & $4.2 \times 10^{-4}$ \\
\hline$n\left(\mathrm{H}_{2}\right)$ & {$\left[\mathrm{cm}^{-3}\right]$} & $7.3 \times 10^{6}$ \\
\hline$n(\mathrm{CO})$ & {$\left[\mathrm{cm}^{-3}\right]$} & $5.7 \times 10^{7}$ \\
\hline$n(\mathrm{OH})$ & {$\left[\mathrm{cm}^{-3}\right]$} & $5.1 \times 10^{3}$ \\
\hline$n(\mathrm{SiO})$ & {$\left[\mathrm{cm}^{-3}\right]$} & $1.3 \times 10^{5}$ \\
\hline$n\left(\mathrm{~N}_{2}\right)$ & {$\left[\mathrm{cm}^{-3}\right]$} & $5.6 \times 10^{6}$ \\
\hline$D$ & {$[\mathrm{kpc}]$} & 0.5 \\
\hline$\rho$ & & 0.1 \\
\hline$T$ & {$[\mathrm{~K}]$} & 2845 \\
\hline$n(\mathrm{H})$ & {$\left[\mathrm{cm}^{-3}\right]$} & $1.1 \times 10^{12}$ \\
\hline$R$ & {$[\mathrm{au}]$} & 2.8 \\
\hline$\tau_{\ell}(232 \mathrm{GHz})$ & & 1.2 \\
\hline$\tau_{\mathrm{c}}(232 \mathrm{GHz})$ & & 1.3 \\
\hline$n(e)$ & {$\left[\mathrm{cm}^{-3}\right]$} & $3.9 \times 10^{7}$ \\
\hline$M_{\mathrm{gas}}$ & {$\left[\mathrm{M}_{\odot}\right]$} & $1.2 \times 10^{-4}$ \\
\hline$n\left(\mathrm{H}_{2}\right)$ & {$\left[\mathrm{cm}^{-3}\right]$} & $5.2 \times 10^{7}$ \\
\hline$n(\mathrm{CO})$ & {$\left[\mathrm{cm}^{-3}\right]$} & $3.0 \times 10^{8}$ \\
\hline$n(\mathrm{OH})$ & {$\left[\mathrm{cm}^{-3}\right]$} & $3.7 \times 10^{4}$ \\
\hline$n(\mathrm{SiO})$ & {$\left[\mathrm{cm}^{-3}\right]$} & $3.1 \times 10^{5}$ \\
\hline$n\left(\mathrm{~N}_{2}\right)$ & {$\left[\mathrm{cm}^{-3}\right]$} & $2.4 \times 10^{7}$ \\
\hline$D$ & {$[\mathrm{kpc}]$} & 2.0 \\
\hline$\rho$ & & 0.1 \\
\hline$T$ & {$[\mathrm{~K}]$} & 2770 \\
\hline$n(\mathrm{H})$ & {$\left[\mathrm{cm}^{-3}\right]$} & $6.0 \times 10^{11}$ \\
\hline$R$ & [au] & 11 \\
\hline$\tau_{\ell}(232 \mathrm{GHz})$ & & 1.3 \\
\hline$\tau_{\mathrm{c}}(232 \mathrm{GHz})$ & & 1.3 \\
\hline$n(e)$ & {$\left[\mathrm{cm}^{-3}\right]$} & $2.0 \times 10^{7}$ \\
\hline$M_{\mathrm{gas}}$ & {$\left[\mathrm{M}_{\odot}\right]$} & $4.2 \times 10^{-3}$ \\
\hline$n\left(\mathrm{H}_{2}\right)$ & {$\left[\mathrm{cm}^{-3}\right]$} & $2.5 \times 10^{7}$ \\
\hline$n(\mathrm{CO})$ & {$\left[\mathrm{cm}^{-3}\right]$} & $1.6 \times 10^{8}$ \\
\hline$n(\mathrm{OH})$ & {$\left[\mathrm{cm}^{-3}\right]$} & $1.8 \times 10^{4}$ \\
\hline$n(\mathrm{SiO})$ & {$\left[\mathrm{cm}^{-3}\right]$} & $2.3 \times 10^{5}$ \\
\hline$n\left(\mathrm{~N}_{2}\right)$ & {$\left[\mathrm{cm}^{-3}\right]$} & $1.4 \times 10^{7}$ \\
\hline
\end{tabular}

Notes. (a) All of the above models satisfy the observed constraints at $232 \mathrm{GHz}: \theta=11 \mathrm{mas}, S_{v}(30 \alpha)=7.3 \mathrm{mJy}$, and and $S_{\mathrm{c}}(232)=7.5 \mathrm{mJy}$.

Such analysis is far beyond the scope of the present work. Even so, it would be interesting for future work to consider that the RTL region can explain the fluxes of prominent forbidden lines in the visible spectrum within an order of magnitude.

Notes. (a) All of the above models satisfy the observed constraints at $232 \mathrm{GHz}$ : $\theta=11$ mas, $S_{v}(30 \alpha)=7.3 \mathrm{mJy}$, and and $S_{\mathrm{c}}(232)=7.5 \mathrm{mJy}$. 
Table D.3. Models reflecting a larger range of parameter space, continued.

\begin{tabular}{lll}
\hline \hline Property & & Primary solution \\
\hline$D$ & {$[\mathrm{kpc}]$} & 1.0 \\
$\rho$ & & 0.1 \\
$T$ & {$[\mathrm{~K}]$} & 2723 \\
$n(\mathrm{H})$ & {$\left[\mathrm{cm}^{-3}\right]$} & $3.68 \times 10^{11}$ \\
$r$ & {$[\mathrm{au}]$} & 8.5 \\
$\tau_{\ell}(232 \mathrm{GHz})$ & & 0.36 \\
$\tau_{c}(232 \mathrm{GHz})$ & & 0.38 \\
$n(e)$ & {$\left[\mathrm{cm}^{-3}\right]$} & $1.2 \times 10^{7}$ \\
$M_{\mathrm{gas}}$ & {$\left[\mathrm{M}_{\odot}\right]$} & $1.2 \times 10^{-3}$ \\
$n\left(\mathrm{H}_{2}\right)$ & {$\left[\mathrm{cm}^{-3}\right]$} & $1.3 \times 10^{7}$ \\
$n(\mathrm{CO})$ & {$\left[\mathrm{cm}^{-3}\right]$} & $9.9 \times 10^{7}$ \\
$n(\mathrm{OH})$ & {$\left[\mathrm{cm}^{-3}\right]$} & $9.4 \times 10^{3}$ \\
$n(\mathrm{SiO})$ & {$\left[\mathrm{cm}^{-3}\right]$} & $1.6 \times 10^{5}$ \\
$n\left(\mathrm{~N}_{2}\right)$ & {$\left[\mathrm{cm}^{-3}\right]$} & $9.1 \times 10^{6}$ \\
\hline$D$ & {$\left[\mathrm{kpc}^{2}\right.$} & 1.0 \\
$\rho$ & & 2.0 \\
$T$ & {$[\mathrm{~K}]$} & 2580 \\
$n(\mathrm{H})$ & {$\left[\mathrm{cm}^{-3}\right]$} & $9.34 \times 10^{10}$ \\
$r$ & {$[\mathrm{au}]^{10}$} & 8.5 \\
$\tau_{\ell}(232 \mathrm{GHz})$ & & 0.39 \\
$\tau_{c}(232 \mathrm{GHz})$ & & 0.40 \\
$n(e)$ & {$\left[\mathrm{cm}^{-3}\right]$} & $2.5 \times 10^{6}$ \\
$M_{\mathrm{gas}}$ & {$\left[\mathrm{M}_{\odot}\right]$} & $6.8 \times 10^{-4}$ \\
$n\left(\mathrm{H}_{2}\right)$ & {$\left[\mathrm{cm}^{-3}\right]$} & $2.5 \times 10^{6}$ \\
$n(\mathrm{CO})$ & {$\left[\mathrm{cm}^{-3}\right]$} & $2.5 \times 10^{7}$ \\
$n(\mathrm{OH})$ & {$\left[\mathrm{cm}^{-3}\right]$} & $1.7 \times 10^{3}$ \\
$n(\mathrm{SiO})$ & {$\left[\mathrm{cm}^{-3}\right]$} & $7.1 \times 10^{4}$ \\
$n\left(\mathrm{~N}_{2}\right)$ & {$\left[\mathrm{cm}^{-3}\right]$} & $2.6 \times 10^{6}$ \\
\hline & & \\
$N$ & &
\end{tabular}

Notes. (a) All of the above models satisfy the constraints at $232 \mathrm{GHz}$ : $\theta=17 \mathrm{mas}, S_{v}(30 \alpha)=7.3 \mathrm{mJy}$, and and $S_{\mathrm{c}}(232)=7.5 \mathrm{mJy}$. 
Table E.1. Weak and forbidden lines at visible wavelengths

\begin{tabular}{lccccccc}
\hline \hline Species & $\begin{array}{c}\lambda_{0}{ }^{a} \\
{[\AA]}\end{array}$ & $\begin{array}{c}v_{\mathrm{LSR}} \\
{\left[\mathrm{km} \mathrm{s}^{-1}\right]}\end{array}$ & $\begin{array}{c}\Delta v^{b} \\
{\left[\mathrm{~km} \mathrm{~s}^{-1}\right]}\end{array}$ & $\begin{array}{c}S_{v, \mathrm{obs}} \\
{[\mathrm{Jy}]}\end{array}$ & $\begin{array}{c}\int S_{v, \mathrm{obs}} \mathrm{d} v \\
{\left[10^{-15} \mathrm{~W} \mathrm{~m}^{-2}\right]}\end{array}$ & $\begin{array}{c}\int S_{v, \text { model }} \mathrm{d} v^{c} \\
{\left[10^{-15} \mathrm{~W} \mathrm{~m}^{-2}\right]}\end{array}$ & $\begin{array}{c}E_{\mathrm{u}}{ }^{d} \\
{\left[\mathrm{~cm}^{-1}\right]}\end{array}$ \\
\hline O I & 5577.339 & $41.3(0.5)$ & $20.7(1.1)$ & $0.81(.04)$ & $0.32(.05)$ & 0.0017 & 33793 \\
O I & 6300.304 & $43.4(0.2)$ & $24.0(0.5)$ & $5.25(.08)$ & $2.13(.05)$ & 0.36 & 15868 \\
O I & 6363.776 & $41.9(0.3)$ & $20.2(0.6)$ & $2.02(.05)$ & $0.68(.03)$ & 0.12 & 15868 \\
Ca II & 7291.469 & $41.5(0.2)$ & $14.7(0.4)$ & $23.40(.40)$ & $5.03(.17)$ & 6.6 & 13711 \\
Ca II & 7323.887 & $41.4(0.1)$ & $17.5(0.2)$ & $18.90(.20)$ & $4.79(.07)$ & 5.0 & 13650 \\
Fe II & 7452.561 & $41.4(0.4)$ & $15.2(0.8)$ & $1.47(.07)$ & $0.32(.02)$ & 0.049 & 15845 \\
Fe I & 7723.207 & $41.4(0.7)$ & $12.7(1.6)$ & $0.60(.07)$ & $0.11(.02)$ & 5.1 & 31323 \\
C I & 8727.126 & $43.1(0.2)$ & $16.3(0.4)$ & $6.50(.13)$ & $1.29(.04)$ & $<0.001$ & 21648 \\
C I & 9850.250 & $39.8(0.8)$ & $21.0(1.9)$ & $0.96(.07)$ & $0.22(.01)$ & $<0.001$ & 10193 \\
& & & & & & & \\
\hline
\end{tabular}

Notes. (a) Wavelength in standard air. (b) FWHM of the Gaussian fit to the line profile. (c) Integrated flux in the reference model, corrected for extinction at $A_{\mathrm{V}}=0.99$ magnitudes (estimated by comparing the observed flux density at $730 \mathrm{~nm}$ with a Kurucz's Atlas 9 grid model for log $g=1.5$ and $T_{\text {eff }}=8500 \mathrm{~K}$ at a distance of $1 \mathrm{kpc}$ ). (d) Energy of the upper state of the transition. References: entries in column 2 come from the NIST Atomic Spectroscopy Database. The numbers in parentheses (...) are the root-mean-square uncertainties derived from the Gaussian fits. 